\title{
Single-molecule studies of conformational states and dynamics in the ABC importer OpuA
}

\author{
Konstantinos Tassis ${ }^{1}$, Ruslan Vietrov ${ }^{1,2}$, Matthijs de Koning ${ }^{1}$, Marijn de Boer ${ }^{1}$, Giorgos \\ Gouridis $^{1,3,4}$ and Thorben Cordes ${ }^{1,5}$ \\ 1 Molecular Microscopy Research Group, Zernike Institute for Advanced Materials, University of Groningen, The Netherlands \\ 2 Department of Biochemistry, Groningen Biomolecular Science and Biotechnology Institute, Netherlands Proteomics Centre \& Zernike \\ Institute for Advanced Materials, University of Groningen, The Netherlands \\ 3 Laboratory of Molecular Bacteriology, Department of Microbiology and Immunology, Rega Institute for Medical Research, KU Leuven, \\ Belgium \\ 4 Structural Biology Division, Institute of Molecular Biology and Biotechnology (IMBB-FORTH), Heraklion-Crete, Greece \\ 5 Physical and Synthetic Biology, Faculty of Biology, Ludwig-Maximilians Universität München, Martinsried, Germany
}

\author{
Correspondence \\ G. Gouridis and T. Cordes, Molecular \\ Microscopy Research Group, Zernike \\ Institute for Advanced Materials, University \\ of Groningen, Nijenborgh 4, Groningen 9747 \\ AG, The Netherlands; Structural Biology \\ Division, Institute of Molecular Biology and \\ Biotechnology (IMBB-FORTH), Nikolaou \\ Plastira 100, Heraklion-Crete, Greece; \\ Physical and Synthetic Biology, Faculty of \\ Biology, Ludwig-MaximiliansUniversität \\ München, Großhadernerstr. 2-4, 82152 \\ Martinsried, Germany \\ Tel: +30 $2810391056(G G) ;+49$ \\ 89218074623 (TC) \\ E-mails: g.gouridis@imbb.forth.gr (GG); \\ cordes@bio.Imu.de (TC)
}

Konstantinos Tassis and Ruslan Vietrov contributed equally to this article

(Received 7 August 2020, revised 2 November 2020, accepted 20 November 2020, available online 6 January 2021)

doi:10.1002/1873-3468.14026
The current model of active transport via $\mathrm{ABC}$ importers is mostly based on structural, biochemical and genetic data. We here establish single-molecule Förster resonance energy transfer (smFRET) assays to monitor the conformational states and heterogeneity of the osmoregulatory type I ABC importer OpuA from Lactococcus lactis. We present data probing both intradomain distances that elucidate conformational changes within the substrate-binding domain (SBD) OpuAC, and interdomain distances between SBDs or transmembrane domains. Using this methodology, we studied ligand-binding mechanisms, as well as ATP and glycine betaine dependences of conformational changes. Our work expands the scope of smFRET investigations towards a class of so far unstudied $A B C$ importers, and paves the way for a full understanding of their transport cycle in the future.

Keywords: ABC transporter; conformational dynamics; membrane transport; osmoregulation; single-molecule Foerster resonance energy transfer; substrate-binding domains
ATP-binding cassette $(\mathrm{ABC})$ transporters represent the most abundant and diverse family of transport proteins known. They play crucial roles in numerous cellular processes including nutrient uptake [1], antibiotic and drug resistance [2], antigen presentation [3], cell volume regulation [4] and others [5-9]. Despite

\section{Abbreviations}

ABC transporters, ATP-binding cassette transporters; SBD, substrate-binding domain; smFRET, single-molecule Förster resonance energy transfer. 
their importance, the majority of molecular models proposed for transport are based on the functional interpretation of static crystal structures, due to the inability of classical biophysical and biochemical techniques to visualize dynamic structural changes [5-9]. Advanced mechanistic insights, for instance knowledge on structural dynamics and heterogeneity of conformational states in transporters, could be beneficial for the fight against pathogenic bacteria [10], and for the treatment of ABC-related diseases such as cystic fibrosis [11] and multidrug resistance in cancer cells [2]. Determining the structural dynamics of drug targets would allow the rational design of high-affinity drugs, and provide a better understanding of a drug's mode of action [12]. However, the development of methods to make a membrane-embedded transport system amenable to complex biophysical investigation represents a huge bottleneck.

Over the past years [13], various laboratories have introduced single-molecule tools to investigate the structural dynamics of active membrane transporters [14-25]. Förster resonance energy transfer in combination with single-molecule detection (smFRET [26-28]) has proven to be a particularly useful tool for the validation of structural models [29-31] and for revealing functional features of transporters, which are mechanistically important, such as conformational heterogeneity [28,32-34]. While the laboratories of Shimon Weiss [14], Scott Blanchard [15-17] and Antoine van Oijen [18] have published the first smFRET studies of secondary active transporters in a detergent environment [14-16] and within liposomes [17,18], we introduced smFRET for studies of $\mathrm{ABC}$ transporters in collaboration with the groups of Bert Poolman, Konstantinos Beis and Robert Tampé. These transporters included importers (GlnPQ [19,20]) and their substrate-binding proteins and domains [21] and the exporter McjD [23]. In addition, we have also studied the non-transporting ABC-protein ABCE1 [35]. Lewinsson [24] and Slotboom [36] have performed detailed smFRET studies on the type II ABC importer BtuCD-F. And the influence of different membrane mimics (detergent, lipid nanodiscs and proteoliposomes) was presented in a recent study on the $\mathrm{ABC}$ exporter MsbA [25]. Advances in the visualization of structural dynamics in transporters have also been made possible by other biophysical techniques such as highspeed AFM [37] and EPR [38,39].

All these studies were motivated by the desire to answer long-standing key questions in the transporter field. These questions include ligand-binding mechanisms, the mechanistic basis of substrate selectivity, the precise timescales of conformational changes in transmembrane and nucleotide binding domains, and the comparative specific conformational states at rest for the distinct type I and type II ABC importers. Furthermore, it remains a goal of researchers in the field to establish complete models of transport that reflect and enhance an understanding of the coordination of transport, ATPase activity and the associated conformational changes. Such models would provide a precise understanding of how substrate-binding and ATP hydrolysis events are coupled and transmitted from one domain to another via conformational changes that ultimately drive substrate transport.

We here extend our smFRET work on ABC transporters to the osmoregulatory OpuA. This importer represents a well-established model system for the type I ABC transporters and is involved in cell volume regulation via the uptake of glycine betaine $[4,40]$. The domain organization of the transporter is shown in Fig. 1A. Poolman and coworkers showed that OpuA is more complex than other type I ABC importers such as the molybdate and maltose permeases because of the presence of two additional cytosolic domains (Cystathionine $\beta$-Synthase; CBS) $[4,40]$. Each homodimer of OpuA consists of two subunits: one composed of the transmembrane domain TMD and substratebinding domain SBD (OpuABC), and the other OpuAA of the nucleotide binding domain (NBD) and CBS in tandem (Fig. 1A). The CBS domains play a role in sensing intracellular ionic strength and, in conjunction with an anionic membrane surface, gate the transporter in response to osmotic stress for import of glycine betaine. The cycling of the NBDs from a dimeric ATP-bound state to a monomeric ADP-state is probably dependent on the CBS-CBS interaction, which is regulated by ionic strength and in particular potassium $[4,40,41]$. The presence of the additional CBS domains might lead to a different mechanism of transport and/or transporter activation than occurs in other type I ABC transporters. OpuA is a paradigm for osmoregulatory $\mathrm{ABC}$ transporters, and the system is widespread in the bacterial and archaeal kingdoms [4]. It is critical for the survival of low GC Gram-positive pathogens under conditions of osmotic stress, but also plays an important role in osmoregulation in Gram-negative pathogenic bacteria [4].

OpuA is a suitable model system for the present work because it can be functionally reconstituted in nanodiscs, where it shows ion- and substrate-dependent ATPase activity with a high coupling efficiency, when nanodiscs are prepared with physiological lipids [40]. In this study, our aims were to monitor by confocal smFRET in solution [19-21,23] various aspects of OpuA conformational dynamics (Fig. 1) and to 

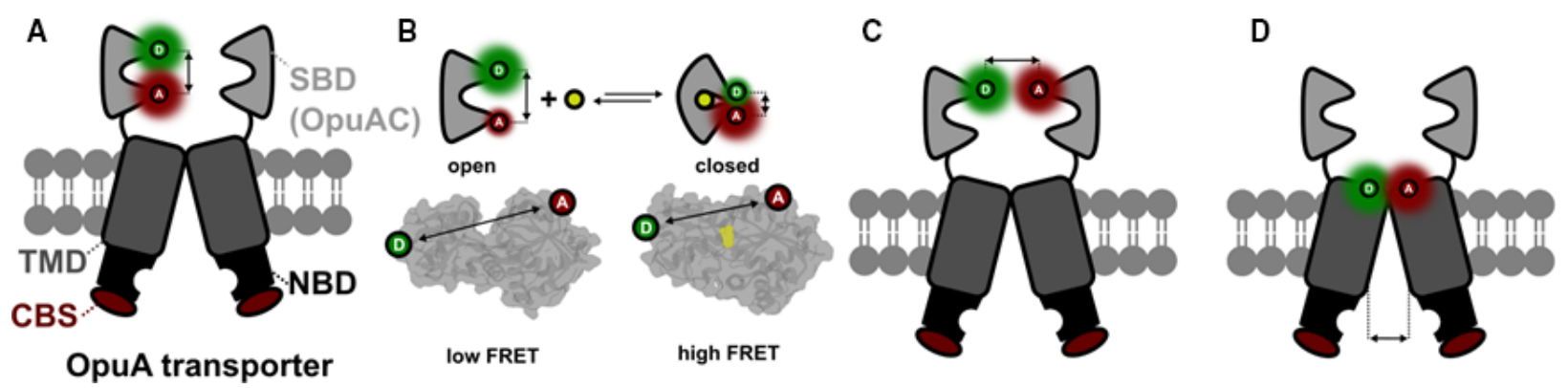

Fig. 1. Setup for smFRET studies of the type I ABC importer OpuA. (A) Structural organization of OpuA and intradomain FRET assay with the SBD. Please note that in our work, the second SBD can also be labelled, but this is not shown for clarity. (B) FRET assay principle to probe the conformational states of the SBD OpuAC via FRET. Open and closed structures are based on the published PBD structures of soluble OpuAC 3L6G and 3L6H, respectively, showing cysteine variant OpuAC (367C/423C) and ligand in yellow. (C, D) Interdomain FRET assays based on (C) SBD and (D) TMD labelling. Reactive coordinates probed in each panel are indicated by arrows and dashed lines.

examine whether any structural heterogeneity exists. We examined the conformational dynamics of the SBD OpuAC in isolation, and in the context of the full transporter, and found that its ligand-binding behaviour was similar (Fig. 1A,B), further supporting the utility of studies on SBDs and SBPs in isolation (as done previously for various ABC-related SBPs/ SBDs [21]). OpuAC showed rapid ligand release times, a feature that might be argued to facilitate high transport rates [40,42-45]. We also studied interdomain interactions between SBDs (Fig. 1C) and evaluated suitable labelling positions for smFRET of the TMDs (Fig. 1D). SBD-SBD interactions were examined in the ligand-free, ATP-bound and glycine betaine-bound states. Finally, for TMD studies, we relied on homology modelling of the TMDs aiming to label the extremes of transmembrane helices on either side of the membrane and generated a large number of cysteine derivatives. These were screened to identify positions with high labelling efficiency and useful biochemical activity. We provide preliminary smFRET data on the most promising OpuA TMD mutant, from which no mechanistic conclusions could be drawn yet.

\section{Methods}

\section{OpuA mutagenesis, expression and membrane vesicle isolation}

The OpuA nucleotide sequence (no endogenous cysteines) from Lactococcus Lactis was subcloned to the pBR322 vector (Addgene, Watertown, MA, USA). The plasmid was used for the introduction of point cysteine mutations eligible for labelling by QC-PCR (Table S2). The point mutants were subsequently cloned in the pNZopuAHis plasmid (Cterminal 6-HIS-tag) using EcoRV-AlnwnI (NEB) for the substrate-binding domain mutants and BamHI-AlwnI
(NEB) for the transmembrane domain mutants (Table S2). For the expression of the mutant proteins, L. lactis Opu401 strain was used, which contains deletion of endogenous OpuA genes from the chromosome. 2- to 5-L cultures were grown anaerobically at $30{ }^{\circ} \mathrm{C}$ in $2 \%(\mathrm{w} / \mathrm{v})$ Gistex LS (Strik BV, Eemnes, the Netherlands) and $200 \mathrm{~mm}$ potassium phosphate $(\mathrm{KPi}), \mathrm{pH} 7.4$, supplemented with $1.0 \%(\mathrm{w} / \mathrm{v})$ glucose and $5 \mu \mathrm{g} \cdot \mathrm{mL}^{-1}$ chloramphenicol. At $\mathrm{OD}_{600}, \sim 2$ the nis $A$ promoter was induced by the addition of $1 \mathrm{ng} \cdot \mathrm{mL}^{-1}$ nisin. Two hours later, the cells were harvested by centrifugation $\left(6000 \mathrm{~g} ; 15 \mathrm{~min} ; 4{ }^{\circ} \mathrm{C}\right)$ and stored at $-20{ }^{\circ} \mathrm{C}$. For the isolation of L. lactis membrane vesicles, all handling was done at $4{ }^{\circ} \mathrm{C}$ unless stated otherwise. The harvested pellet was resuspended in $50 \mathrm{~mm} \mathrm{KPi}, 200 \mathrm{~mm} \mathrm{KCl}, 20 \%$ glycerol, pH 7.4 (buffer A) in the presence of $1.5 \mathrm{~mm}$ dithiothreitol (DTT). To reduce the viscosity caused by the release of DNA, $100 \mu \mathrm{g} \cdot \mathrm{mL}^{-1}$ DNase and $2 \mathrm{~mm} \mathrm{MgSO}_{4}$ were added. The suspension was disrupted through a continuous disruption cycle, twice at 40 Psi in a Constant Cell Disruption System LTD (Daventry, UK). In the disrupted material, $1 \mathrm{~mm}$ PMSF and $5 \mathrm{~mm}$ EDTA, $\mathrm{pH}$ 8.0, were added. First, large cellular debris were removed by centrifugation $\left(11800 \mathrm{~g} ; 20 \mathrm{~min} ; 4^{\circ} \mathrm{C}\right)$, the pellet was discarded, and the supernatant was centrifuged again (125000 $\mathrm{g}$; $60 \mathrm{~min} ; 4^{\circ} \mathrm{C}$ ). The pellet containing membrane vesicles was resuspended in buffer A and $1 \mathrm{~mm}$ DTT and centrifuged again $\left(125000 \mathrm{~g} ; 60 \mathrm{~min} ; 4^{\circ} \mathrm{C}\right)$ to remove any remaining soluble components. The isolated membrane vesicles were resuspended in buffer A in the presence of $1 \mathrm{~mm}$ DTT, aliquoted and flash-frozen in liquid $\mathrm{N}_{2}$ and then stored in $-80{ }^{\circ} \mathrm{C}$. The total protein concentration was determined using the Pierce ${ }^{\mathrm{TM}}$ BCA Protein Assay Kit (Fisher Scientific, Landsmeer, Netherlands).

\section{OpuA purification}

All the handling described below was done at $4{ }^{\circ} \mathrm{C}$ unless mentioned otherwise. The stored membrane 
vesicles were thawed and resuspended in buffer A (50 mM KPi, $200 \mathrm{~mm} \mathrm{KCl,} \mathrm{20 \%} \mathrm{glycerol,} \mathrm{pH} \mathrm{7.4)} \mathrm{with}$ the addition of $1 \mathrm{~mm}$ DTT and $10 \mathrm{~mm} n$-Dodecyl $\beta$-Dmaltoside (DDM) and incubated for $60 \mathrm{~min}$ while gently agitated. The solubilized protein was separated from any insolubilized material by centrifugation $\left(267000 \mathrm{~g} ; 20 \mathrm{~min} ; 4{ }^{\circ} \mathrm{C}\right.$ ). The harvested supernatant was diluted with buffer A to a final DDM concentration of $2 \mathrm{~mm}$ and loaded to $\mathrm{Ni}^{2+}$-Sepharose ${ }^{\mathrm{TM}} 6$ fast flow resin (GE Healthcare, Hoevelaken, Netherlands already equilibrated with $10 \mathrm{CV}$ buffer A with $780 \mu \mathrm{M}$ DDM) and was incubated for 60 min under gentle agitation. The resin-bound material was washed three consecutive times $(10 \mathrm{CV}$ of each: buffer A with $1 \mathrm{~mm}$ DTT and $780 \mu \mathrm{M}$ DDM, buffer A with $1 \mathrm{~mm}$ DTT and $20 \mathrm{~mm}$ imidazole and $780 \mu \mathrm{M}$ DDM, and buffer A with $1 \mathrm{~mm}$ DTT and $40 \mathrm{~mm}$ imidazole and $780 \mu \mathrm{M}$ DDM) to remove all the weakly bound proteins. The purified protein was then eluted with buffer A with $200 \mathrm{~mm}$ imidazole and $780 \mu \mathrm{M}$ DDM.

\section{OpuA nanodisc formation and purification}

An optimized synthetic lipid mixture dissolved in $50 \mathrm{~mm} \mathrm{KPi}$, pH 7.0 [50\% 1,2-dioleoyl-sn-glycero-3-phosphoethanolamine, DOPE: 12\% 1,2-dioleoyl-sn-glycero-3phosphocholine, DOPC: 38\% 1,2-dioleoyl-sn-glycero-3phospho-(1'-rac-glycerol), DOPG, Avanti Polar Lipids, Alabaster, AL, USA] was prepared as described [40]. The lipid mixture was thawed and subsequently extruded through a $400-\mathrm{nm}$ pore size polycarbonate filter, generating large unilamellar vesicles. $12 \mathrm{~mm}$ DDM was added, and the mixture was vortexed until optically clear. The final reconstitution reaction was $50 \mathrm{~mm} \mathrm{KPi}$, $20 \%$ glycerol, $12 \mathrm{~mm}$ DDM, and contained $1 \mathrm{nmol}$ OpuA (labelled/unlabelled), $10 \mathrm{nmol} \mathrm{MSP}_{1} \mathrm{D}_{1}, 1 \mu \mathrm{mol}$ lipids in a final volume of $700 \mu \mathrm{L}$. To control for size and formation of nanodiscs, reconstitution reactions without OpuA were carried out and tested using sizeexclusion chromatography (SEC). The reaction was incubated for $60 \mathrm{~min}$ at $4{ }^{\circ} \mathrm{C}$, while gently agitated. Then, $500 \mathrm{mg}$ of SM2 biobeads was added to the reaction volume and incubated for $1-12 \mathrm{~h}$ with best result at $1-2 \mathrm{~h}$ of incubation. The biobeads were removed, and the solution was centrifuged $(18000 \mathrm{~g} ; 10 \mathrm{~min}$; $4{ }^{\circ} \mathrm{C}$ ) to precipitate any aggregated lipids and proteins. To determine the composition of the nanodiscs and to separate the nanodiscs from aggregates and empty nanodiscs, the supernatant from the previous step was purified using SEC, using a Superdex $200 \quad 10 / 300$ GL column (GE Healthcare) that was previously equilibrated with $50 \mathrm{~mm} \mathrm{KPi,} \mathrm{pH} \mathrm{7.0,} 200 \mathrm{~mm} \mathrm{KCl}$ and $4 \%$ $\mathrm{w} / \mathrm{v}$ glycerol buffer. The protein composition of the SEC fractions was verified by SDS/PAGE on $12 \%$ polyacrylamide gels.

\section{$M S P_{1} D_{1}$ expression and purification}

Membrane scaffold protein $\mathrm{MSP}_{1} \mathrm{D}_{1}$ was used for the formation of nanodiscs [40]. Escherichia coli BL21(DE3) cells were freshly transformed with the $\mathrm{pMSP}_{1} \mathrm{D}_{1}$ plasmid and grown in $2 \mathrm{~L}$ Terrific Broth-kanamycin $\left(10 \mu \mathrm{g} \cdot \mathrm{mL}^{-1}\right)$ medium at $37{ }^{\circ} \mathrm{C}$ under aerobic conditions. At $\mathrm{OD}_{600}$ of 1.5 , the culture was induced with $1 \mathrm{~mm}$ isopropyl 1-thio-Dgalactopyranoside (IPTG); $3 \mathrm{~h}$ later, the cells were harvested by centrifugation $\left(8000 \mathrm{~g} ; 20 \mathrm{~min} ; 4^{\circ} \mathrm{C}\right)$. From this step onwards, all the processes were done at $4{ }^{\circ} \mathrm{C}$ and all solutions were at $4{ }^{\circ} \mathrm{C}$, unless stated otherwise. The cell pellet was resuspended in $20 \mathrm{~mm} \mathrm{KPi,} 1 \%$ Triton X-100, $1 \mathrm{~mm}$ PMSF at $\mathrm{pH}$ 7.4. The cells were lysed by sonification ( $5 \mathrm{~s}$ on $/ 5 \mathrm{~s}$ off, $70 \%$ amplitude, $3 \mathrm{~min}$ ). The cell lysate was fractionated by centrifugation $\left(125000 \mathrm{~g} ; 75 \mathrm{~min} ; 4{ }^{\circ} \mathrm{C}\right)$, and the pellet was discarded. $\mathrm{Ni}^{2+}$-Sepharose ${ }^{\mathrm{TM}} 6$ fast flow resin (GE Healthcare) was equilibrated with 10 column volumes of $40 \mathrm{~mm} \mathrm{KPi,} \mathrm{pH} \mathrm{7.4,} \mathrm{and} \mathrm{the} \mathrm{supernatant} \mathrm{of} \mathrm{the}$ previous centrifugation was gravity loaded to the column. The resin-bound $\mathrm{MSP}_{1} \mathrm{D}_{1}$ was sequentially washed with 10 column volumes of $40 \mathrm{~mm}$ Tris $/ \mathrm{HCl}, 0.3 \mathrm{~m} \mathrm{NaCl}, 1 \%$ Triton $\mathrm{X}-100, \mathrm{pH} 8.0$, then $40 \mathrm{~mm}$ Tris $/ \mathrm{HCl}, 0.3 \mathrm{M} \mathrm{NaCl}$, $50 \mathrm{~mm}$ sodium cholate, $20 \mathrm{~mm}$ imidazole, $\mathrm{pH} 8.0$, and lastly with $40 \mathrm{~mm}$ Tris $/ \mathrm{HCl}, 0.3 \mathrm{~m} \mathrm{NaCl}, 50 \mathrm{~mm}$ imidazole, $\mathrm{pH}$ 8.0. $\mathrm{MSP}_{1} \mathrm{D}_{1}$ was then eluted with $40 \mathrm{~mm}$ Tris $/ \mathrm{HCl}$, $0.3 \mathrm{M} \mathrm{NaCl}, 0.4 \mathrm{~m}$ imidazole, $\mathrm{pH}$ 8.0. The eluent was dialysed overnight (SnakeSkin ${ }^{\mathrm{TM}}$ Dialysis Tubing, 7K MWCO Fisher Scientific) against $20 \mathrm{~mm}$ Tris $/ \mathrm{HCl}, 0.1 \mathrm{M} \mathrm{NaCl}$, $0.5 \mathrm{~mm}$ EDTA, pH 7.4. Lastly, it was dialysed once more overnight against $20 \mathrm{~mm}$ Tris $/ \mathrm{HCl}, 0.1 \mathrm{M} \mathrm{NaCl}, 0.5 \mathrm{~mm}$ EDTA, $50 \%$ glycerol, $\mathrm{pH}$ 7.4. The protein concentration was calculated by absorbance at $280 \mathrm{~nm}$ (extinction coefficient $21000 \mathrm{M}^{-1} \cdot \mathrm{cm}^{-1}$; see Fig. S5a). The purified protein was aliquoted and stored at $-20{ }^{\circ} \mathrm{C}$.

\section{ATPase activity assay}

To determine the activity of the reconstituted OpuA, a coupled-enzyme activity assay was used to measure the ATPase activity of the reconstituted transporter. The coupled enzymatic reaction contained $\sim 2$ units of pyruvate kinase/lactic dehydrogenase (rabbit muscle isolate mixture; SigmaAldrich, Zwijndrecht, Netherlands) $50 \mathrm{~mm} \mathrm{KPi,} \mathrm{pH} \mathrm{7.0,}$ $300 \mathrm{~mm}$ NADH, $4 \mathrm{~mm}$ phosphoenolpyruvate (PEP), $62 \mu \mathrm{M}$ glycine betaine, $300 \mathrm{~mm} \mathrm{KCl}$, and $4 \mu \mathrm{g}$ of OpuA reconstituted in nanodiscs. The change in absorbance at $340 \mathrm{~nm}$ was observed with a Synergy MX 96-well plate reader (Bio Tek Instruments, Inc., Winooski, VT, USA). The reaction components were incubated in the plate reader at $30{ }^{\circ} \mathrm{C}$ for 3 min. Then, $10 \mathrm{~mm} \mathrm{MgATP,} \mathrm{pH} \mathrm{7.0,} \mathrm{was} \mathrm{added} \mathrm{shaking}$ the plate for $10 \mathrm{~s}$ to mix the compounds. A 7-min kinetic read $\left(340 \mathrm{~nm}, 30^{\circ} \mathrm{C}\right)$ with minimal time intervals was executed. The data were corrected for the path length of each individual reaction volume. For every mutant tested, 
controls were done in the absence of: (a) glycine betaine, (b) $\mathrm{KCl}$, (c) glycine betaine and $\mathrm{KCl}$ (Fig. S6). The ATPase activity was calculated from the slope of the measurements and normalized against the WT internal control.

\section{Radiolabelled isotope uptake}

To monitor transport activity, we used an in vivo-radiolabelled isotope uptake assay in L. lactis according to a previously published method [46]. Briefly, L. lactis Opu401 transformed with the appropriate plasmid was grown in glucose-CDM with $5 \mu \mathrm{g} \cdot \mathrm{mL}^{-1}$ chloramphenicol. At $\mathrm{OD}_{600}$ $\sim 0.5$, the culture was induced with $0.01 \%(\mathrm{v} / \mathrm{v})$ nisin A (filter-sterilized culture supernatant from L. lactis NZ9700) until $\mathrm{OD}_{600} \sim 1$. The cells were washed and resuspended at $2.5 \mathrm{mg}$ of cell protein $\cdot \mathrm{mL}^{-1}$ in $50 \mathrm{~mm}$ HEPES-methylglucamine $\mathrm{pH}$ 7.3. Next, $10 \mathrm{~mm}$ glucose was added, and the cells were incubated for $5 \mathrm{~min}$ at $30^{\circ} \mathrm{C}$. To start the uptake, a final concentration of $600 \mathrm{~mm}$ sucrose (osmotic stress to increase the internal ionic strength of the cell and activate $\mathrm{OpuA}), 1 \mathrm{~mm}\left[{ }^{14} \mathrm{C}\right]$ glycine betaine in the presence and $50 \mu \mathrm{g} \cdot \mathrm{mL}^{-1}$ chloramphenicol (to prevent protein synthesis) were added to a reaction volume of $500 \mu \mathrm{L}$. Eighty microlitre samples were taken at regular time intervals and diluted with $2 \mathrm{~mL}$ of ice-cold assay buffer of equal osmolality and filtered through $0.45-\mu \mathrm{m}$ cellulose nitrate filters under high vacuum. The membranes were washed with icecold $50 \mathrm{~mm}$ HEPES/methylglucamine, $\mathrm{pH}$ 7.3. After drying, $2 \mathrm{~mL}$ of scintillating liquid was used to dissolve the membranes. The radioactivity was determined in a scintillation counter.

\section{Fluorophore labelling of OpuA}

OpuAC was labelled as described previously [21]. For the labelling process of OpuA, the final elution step was eschewed during purification and the resin was drained and washed with buffer A and $780 \mu \mathrm{M}$ DDM to remove DTT. Then, $100 \mathrm{nmol}$ of maleimide fluorophores (molar ratio of 7-10 fluorophores per cysteine available) were solubilized in $10 \mu \mathrm{L}$ water-free dimethyl sulfoxide (DMSO) at room temperature and then suspended in buffer A and $780 \mu \mathrm{M}$ DDM. The fluorophore mixture was added to the drained resin and incubated for $60 \mathrm{~min}$ under gentle agitation. The excess of fluorophores was washed with $10 \mathrm{CV}$ buffer $\mathrm{A}$ and $780 \mu \mathrm{M}$ DDM, and the labelled OpuA was eluted with buffer A, $200 \mathrm{~mm}$ imidazole, $780 \mu \mathrm{M}$ DDM (Fig. S5b,c). The protein and fluorophore concentrations were estimated using Lambert-Beer's law $(A=\varepsilon l c)$. The absorbance was calculated by measurements at 280, 560 and $655 \mathrm{~nm}$ (calculating the area under the chromatogram of the relevant fraction). The path length was $0.1 \mathrm{~cm}$, and the extinction coefficients were available in the data sheets of the fluorophores (Table S4). Labelling efficiency was calculated as $100 \times$ moles of total fluorophores/moles of cysteines. The purified OpuA was directly used for reconstitution into nanodiscs.

This final protocol was obtained after we observed that labelling efficiencies dropped significantly after the reconstitution step, as we were determining the labelling efficiency of our sample prior and subsequent to the reconstitution in bilayer nanodiscs. After many trials, we could attribute this effect to be dependent on the use of SM2 biobeads. Biobead systems have been used in the past to scavenge unbound rhodamine dyes [47]. To better control labelling efficiency and sticking of protein mediated via fluorophores to the biobeads, we used MalE as test system, for which we obtained high labelling efficiency in variant T36C/S352C [21]. To see the effect of the SM2 biobeads on a soluble protein; $2.5 \mathrm{nmol}$ of MalE (T36C/S352C) was subjected to size-exclusion chromatography to determine the baseline amount of protein in absorbance units (same buffers as used for OpuA). $2.5 \mathrm{nmol}$ of protein was then incubated with $1000 \mathrm{mg}$ of SM2 biobeads (the reaction volume was doubled to $1.4 \mathrm{~mL}$ ) overnight at $4{ }^{\circ} \mathrm{C}$ (in reconstitution buffer without lipids, detergent and $\mathrm{MSP}_{1} \mathrm{D}_{1}$ ). After incubation, the SM2 biobeads were separated from the solution by centrifugation and the supernatant was subjected to SEC. The chromatogram (Fig. S9b) was corrected for the amount of supernatant lost during the separation of the SM2 biobeads from the protein solution. Then, it was normalized against the absorbance of nontreated MalE (Fig. S9a). After extended incubation times, the SM2 biobeads can adsorb soluble proteins as indicated by a loss of $\sim 35 \%$ of unlabelled MalE (T36C/S352C); Fig. S9. To further test our hypothesis, $5 \mathrm{nmol}$ of MalE (T36C/S352C) was labelled as described above with the only difference to omitting DDM. We used fluorophores Alexa Fluor 555 (Ax555, $50 \mathrm{nmol})$ and Alexa Fluor 647 (Ax647, $50 \mathrm{nmol}$ ) to achieve a molar ratio of $10: 1$ regarding fluorophore and cysteine. The eluted labelled protein sample was then split in two parts. Half of it was analysed by SEC to determine the labelling efficiency $(>75 \%)$ and also provide a baseline (Fig. S9c). The other half was incubated with $1000 \mathrm{mg}$ SM2 biobeads (1.4 $\mathrm{mL}$ reaction volume) for $3 \mathrm{~h}$ at $4{ }^{\circ} \mathrm{C}$ (in reconstitution buffer without lipids, detergent and $\mathrm{MSP}_{1} \mathrm{D}_{1} ; \quad$ Fig. S9c). After the incubation, MalE (T36C/S352C) was separated from the SM2 biobeads and again analysed by size-exclusion chromatography. We observed a dramatic reduction in retrievable labelled protein (Fig. S9d). Based on these results, further optimization was conducted, in which we reduced the duration of the nanodisc formation reaction to $1-2 \mathrm{~h}$ as described above with the best labelling results observed at $1 \mathrm{~h}$.

\section{OpuA structure modelling}

The homology model for the prediction for candidate mutagenic sites was done using Swiss Model Server $[48,49]$. The template structure used was the molybdate/ 
tungstate $\mathrm{ABC}$ transporter from Archaeoglobus fulgidus (ModBC, 2ONK), the Maltose transport system from E. coli (MalG, 2R6G and 4JBW), the molybdate/tungstate transporter from Methanosarcina acetivorans (ModBC, 3D31) and the methionine importer (MetNI, 3DHW). The sequence identity between $\mathrm{OpuA}$ and the templates was between $20.4 \%$ and $26.6 \%$, while similarity was $\sim 30 \%$. To calculate the relative distant change, the produced model was aligned with the inward- and outward-facing conformations of the maltose transporter in PYMOL [50] and the distances were measured also with PYMOL for $\mathrm{Ca}$ positions of the residues.

\section{Solution smFRET measurements and data analysis}

The methods and experimental devices used here have been described previously in detail $[19,20,51,52]$. In brief, labelled OpuAC or OpuA was diluted to $20-100$ pm in imaging buffer: $50 \mathrm{~mm}$ potassium phosphate $\mathrm{pH} 7.4,1 \mathrm{~mm}$ Trolox and $10 \mathrm{~mm}$ cysteamine (pH 7.5; Sigma-Aldrich). Previously, the cover slip (no. $1.5 \mathrm{H}$ precision cover slides, VWR, Marienfeld, Amsterdam, Netherlands) was passivated with a BSA solution $\left(200 \mu \mathrm{L}\right.$ of $1 \mathrm{mg} \cdot \mathrm{mL}^{-1}$ BSA) for $30 \mathrm{~s}$ to minimize fluorophore interactions with the glass slide. The measurements were done using a custom-built confocal microscope $[19,20]$ at room temperature. Excitation was at 532 and $640 \mathrm{~nm}$ in accordance with the fluorophore absorbance maxima (SuperK Extreme, NKT Photonics A/S, Birkerød, Denmark). Alternation between the two excitation wavelengths was achieved by $50 \mu$ s alternation. The output beam was coupled to a single-mode fibre (PM-S405-XP; Thorlabs LTD, Ely, UK) and recollimated (MB06; Qioptiq Photonics GmbH \& Co. KG, Goettingen, Germany) before entering an oil immersion objective $(60 \times$, NA 1.35 , UPLSAPO 60XO; Olympus Germany GmbH, Düsseldorf, Germany). Excitation and emission were separated by a dichroic beam splitter (zt532/642rpc; AHF analysentechnik AG, Tübingen, Germany) mounted in an inverse microscope body (IX71; Olympus Germany GmbH). Fluorescence emitted by diffusing molecules in solution was collected by the same oil objective, focussed onto a $50 \mathrm{~nm}$ pinhole and spectrally separated (640DCXR; AHF analysentechnik AG) onto two APDs $(\tau$-spad, $<50$ dark counts $\cdot \mathrm{s}^{-1}$; Picoquant, Berlin, Germany) with the appropriate spectral filtering (donor channel: HC582/75; acceptor channel: Edge Basic 647LP; both AHF analysentechnik AG). Unless mentioned otherwise in the figure legends ALEX data $[23,51]$ were analysed with a dual-colour burst search $(M=15, T=500 \mu$ s and $L=25)$ and the resulting data were plotted with $61 \times 61$ bins considering an S-range of 0.3-0.8 with additional thresholding of all photons $>100$. The data of OpuA (A12C) were collected by a similar confocal microscopy setup as described in Ref. [51]. Here, the excitation was done via two Coherent OBIS lasers centred at 532 and $637 \mathrm{~nm}$ and the objective lens was $60 \times$, NA 1.2, UPlanSAPO $60 \mathrm{XO}$ (Olympus Germany $\mathrm{GmbH})$.

\section{Surface scanning microscopy and data analysis}

For surface immobilization of OpuA nanodiscs, custom flow cells were made as previously described in Refs $[19,23,52]$. The flow cell surface was functionalized at room temperature with a neutravidin solution; $0.2 \mathrm{mg} \cdot \mathrm{mL}^{-1}$ neutravidin (Invitrogen, Carlsbad, CA, USA) in $50 \mathrm{~mm}$ potassium phosphate $\mathrm{pH} 7.4$, filtered with $250-\mu \mathrm{m}$ syringe filter (buffer B) for 5-10 min. The unbound excess of neutravidin was washed with the same buffer. Subsequently, the surface of the flow cell was incubated with an anti-His antibody (in buffer B) for $5 \mathrm{~min}$ and washed again with buffer B. Then, His-tag containing OpuAC was introduced to the flow cell (in buffer B supplemented with $10 \mathrm{~mm}$ of $( \pm$ )6-Hydroxy2,5,7,8-tetramethylchromane-2-carboxylic acid (Trolox; Sigma-Aldrich Chemie N.V. Zwijndrecht, Netherlands) as a photostabilizer [20]) and incubated for 30-120 s while at the same time scanning the surface to determine the optimum density. When the particle density was adequate, the excess of labelled proteins was washed away, using buffer $\mathrm{B}$ with $10 \mathrm{~mm}$ Trolox. Fluorescence traces were recorded at room temperature. The fluorescent trajectories were analysed using a hidden Markov Model [53] as described in Ref. [21]. The binning time was $1.5 \mathrm{~ms}$.

\section{Results}

To determine whether isolated OpuAC retains native ligand-binding behaviour, we compared the isolated SBD (Fig. 1B) with the SBD present in full-length OpuA (Fig. 1A). To this end, we generated two double cysteine derivatives of OpuAC: OpuAC (360C-423C) also used in Ref. [21] and OpuAC (367C/423C). Mutations were located on the rigid subdomains of OpuAC, and substrate binding was expected to increase the proximity of donor and acceptor fluorophores on labelled OpuAC, that is lead to a high FRET efficiency state upon ligand binding (Fig. 1B). Our goal initially was to maximize the FRET efficiency change during the conformational motion, and so the impact of mutations or fluorophore labelling on OpuAC's ability to dock with the transporter was not considered.

\section{Conformational dynamics of isolated OpuAC}

To characterize the conformational dynamics of soluble OpuAC labelled with Cy3b and ATTO647N, we used both alternating laser excitation (ALEX) on diffusing molecules and confocal scanning microscopy on 
surface-immobilized ones [21]. In agreement with previously published results [21], OpuAC (360C/423C) changed its conformation in a ligand-dependent fashion (Fig. 2A-C).

Both the open-unliganded (low FRET) and closedliganded states (high FRET) were detectable, and their occupancy changed as a function of the glycine betaine concentration in the buffer solution (Fig. 2A,B). In the absence of glycine betaine, we find exclusively occupation of the open state (Fig. 2A,B - apo), as was also shown previously [21]. This result and its interpretation were valid for freely diffusing (Fig. 2A) and surface-immobilized OpuAC (Fig. 2B). Identical results were obtained when an alternative pair of fluorophores was used for labelling (Alexa Fluor 555, Alexa Fluor 647; Fig. S1). This suggests that the fluorophores do not compromise the conformational changes of OpuAC and do not alter the ligand-binding affinity. The supporting data in Fig. S1 also show the two-dimensional character of the ALEX experiments, that is low stoichiometry acceptor-only molecules $(S<0.3)$, intermediate stoichiometry donor-acceptor-labelled molecules $(0.3<\mathrm{S}<0.8)$ and high stoichiometry donor-only molecules $(\mathrm{S}>0.8)$. For histograms shown in Fig. 2A and subsequent figures where $1 \mathrm{D} \mathrm{E}^{*}$ histograms are presented, we focussed on the analysis of the FRET efficiency distributions in the intermediate stoichiometry region, where the protein carries one donor and one acceptor fluorophore.

Next, we studied the dynamic conversion between conformational states (Fig. 2C). For this, the temporal evolution of donor and acceptor fluorescence signals of surface-immobilized (as described in Surface microscopy section in Methods) OpuAC molecules was followed using confocal scanning microscopy. The FRET efficiency time traces show frequent switching between the low and high FRET state, which indicates the opening and closing transitions of OpuAC (Fig. 2B). To obtain the associated kinetics, the time traces were fitted with a two-state hidden Markov model (HMM). The lifetime distributions of the low and high FRET states were obtained from the fit (as described in Ref.
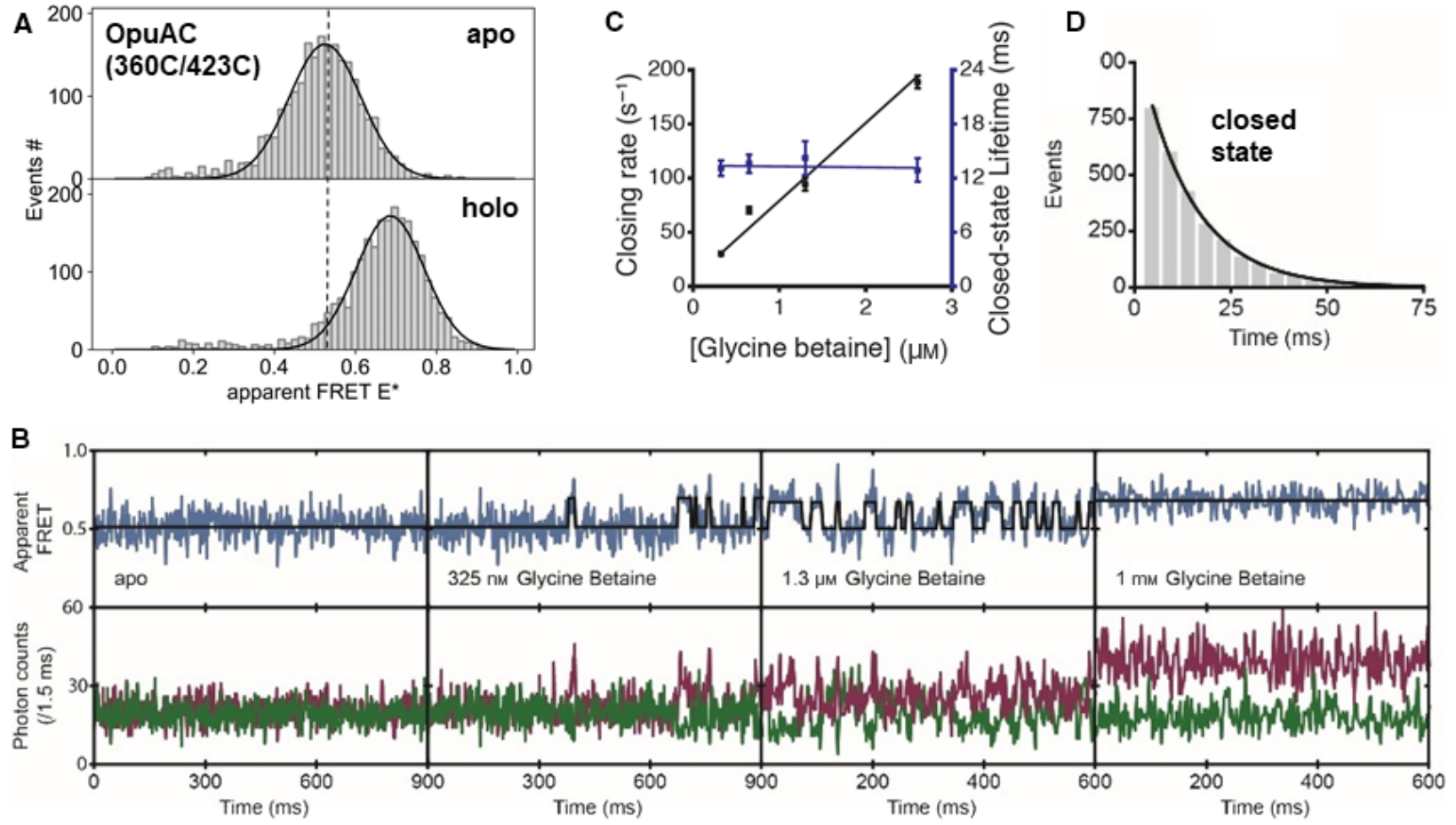

Fig. 2. smFRET studies of isolated OpuAC (360C-423C) with Cy3B and ATTO647N as donor and acceptor fluorophores, respectively. (A) Apparent FRET efficiency histogram of freely diffusing fluorophore-labelled OpuAC molecules, obtained from the solution-based smFRET and ALEX measurements under the indicated conditions. (B) Fluorescence trajectories of OpuAC under different conditions as indicated; donor (green) and acceptor (red) photon counts were binned with $1.5 \mathrm{~ms}$. The top panel shows calculated apparent FRET efficiency (blue) with the most probable state trajectory of Hidden Markov Model (HMM) (black). (C) Average closing rate (black) and lifetime of the closed state (purple) as function of glycine betaine concentration. Error bars indicate the 95\% confidence interval. (D) Lifetime distribution of the closed state as obtained from the most probable state trajectory of the HMM of all molecules. Grey bars are the binned data, and the solid line is an exponential fit. 
[21]; Fig. 2C,D). From this, we extracted the average opening and closing rates (Fig. 2C,D). We observed that the average opening rate was largely concentration-independent, whereas the average closing rate scales approximately linearly with glycine betaine concentration. This ligand dependency is indicative of an induced-fit ligand-binding mechanism [19-21,23], yet the limited time resolution of $1.5 \mathrm{~ms}$ cannot rule out faster conformational switching under the conditions tested. We stress, however, that the fluorescent time traces of OpuAC (360C/423C) are of very good quality and $\mathrm{OpuAC}$ has the fastest ligand release time that we have detected with HHM analysis and dwell time analysis amongst all SBPs and SBDs that we have analysed [21]. Our data (see below) also suggest that the ligandbinding affinity $\left(K_{\mathrm{d}}\right)$ was in the low micromolar range (Fig. 2C), as determined by titration experiments on freely diffusing molecules. This ligand affinity was in agreement with a value from published bulk experiments where a $K_{\mathrm{d}}$-value of $\sim 4 \mu \mathrm{M}$ was reported [54].

\section{Conformational states and dynamics of soluble and membrane-anchored OpuAC}

In order to quantify the ligand affinity of free OpuAC, in comparison with the physiological situation, where the protein is covalently tethered to the OpuA translocator (TMDs), we performed a glycine betaine titration (Fig. 3). For this, we used OpuAC (367C/423C) with a slightly altered labelling scheme as compared to the data presented in Fig. 2, that is OpuAC (360C/ 423C). The results were qualitatively similar, and only the absolute FRET efficiencies were distinct between the experiments due to differences in the intercysteine distances (compare apo/holo in Figs 2A and 3A). By fitting the FRET efficiency histograms with two Gaussian distributions, the relative populations of the open and closed states were obtained as function of glycine betaine concentration [GB] (Fig. 3B). The data points were fit to the function $r_{\mathrm{c}}=[\mathrm{GB}] /\left(K_{\mathrm{d}}+[\mathrm{GB}]\right)$, which results in a dissociation constant $K_{\mathrm{d}}$ of $\sim 6 \mu \mathrm{M}$ for free OpuAC (95\% confidence interval); the fit is displayed as a black line in Fig. 3B. In the equation, $r_{\mathrm{c}}$ is the fraction of OpuAC in the closed state.

To study the conformational switching for OpuAC within the whole transporter, we reconstituted the corresponding cysteine variant of OpuA $(367 \mathrm{C} / 423 \mathrm{C})$ into nanodiscs using previously established protocols (for details, see Methods) [40]. The experimental procedure is schematically sketched in Fig. 4. In brief, OpuA derivatives were expressed and purified as described previously [40] (Fig. S4). The cell pellets were disrupted and cell lysates fractionated by centrifugation to obtain membrane vesicles containing the overexpressed L. lactis OpuA derivatives (Fig. 4, V). OpuA was solubilized with DDM and immobilized on a $\mathrm{Ni}^{2+}$-sepharose ${ }^{\mathrm{TM}}$ resin (Fig. 4, I). Fluorophore labelling was performed at this stage. After removal of excess dye, OpuA was reconstituted into nanodiscs. The nanodiscs were purified by size-exclusion chromatography (SEC) (Fig. S4). Selected fractions from SEC were analysed by SDS/PAGE to verify that the transporter complex was reconstituted intact (Fig. S4). Proper biochemical activity was verified by assessing the glycine betaine and potassium-induced ATPase activity, which was stimulated fourfold to 10 -fold over the basal activity (Fig. S5, wt OpuA).

On SEC, we obtained a peak eluting at $\sim 12 \mathrm{~mL}$ containing the reconstituted Nd-OpuA (367C/423C) derivative [40] and a second one with the empty nanodiscs at $\sim 14 \mathrm{~mL}$ (Fig. 3C). By determining the absorbance at the indicated wavelengths in the corresponding elution volumes (grey bar, Fig. 3C), we estimated the labelling efficiencies, that is the relative concentration of donor and acceptor dye to OpuA (for details, see Methods). As expected, from the size difference between free and nanodisc-embedded OpuAC within the entire transporter, the nanodisc-reconstituted OpuA shows a slightly shifted burst length distribution with respect to free OpuAC, indicating slower diffusion (Fig. 3D).

Next, we performed smFRET on nanodisc-reconstituted Nd-OpuA (367C/423C) (Fig. 3E) at different ligand concentrations, as previously done for free OpuAC (Fig. 3A). OpuA can be labelled with more than two fluorophores in the $367 \mathrm{C} / 423 \mathrm{C}$ variant, since four cysteines are present due to the fact that each protomer contains an OpuAC SBD. To exclude interdomain artefacts in our smFRET experiments, we analysed only molecules with a stoichiometry value $>0.5$ to bias our analysis towards OpuA molecules bearing fewer fluorophores. This selection was based on the analysis of photon counting histograms (Fig. S3) of Nd-OpuA (367C/423C). We noted that the acceptor-based acceptor emission AA was lower in the high stoichiometry region, which suggests fewer acceptor labels here. Furthermore, low S molecules also show stronger donor-quenching, which is likely due to the presence of multiple acceptor fluorophores.

In line with this interpretation and data selection, the resulting FRET efficiency histograms of soluble OpuAC and Nd-OpuA (367C/423C) were similar. In addition, the fit of the glycine betaine response suggests a $K_{\mathrm{d}}$-value of $\sim 2 \mu \mathrm{m}$ for $\mathrm{Nd}-\mathrm{OpuA}(367 \mathrm{C} / 423 \mathrm{C})$ (Fig. 3B, grey fit line), which is threefold higher as compared to the soluble OpuAC $(\sim 6 \mu \mathrm{M})$. This 

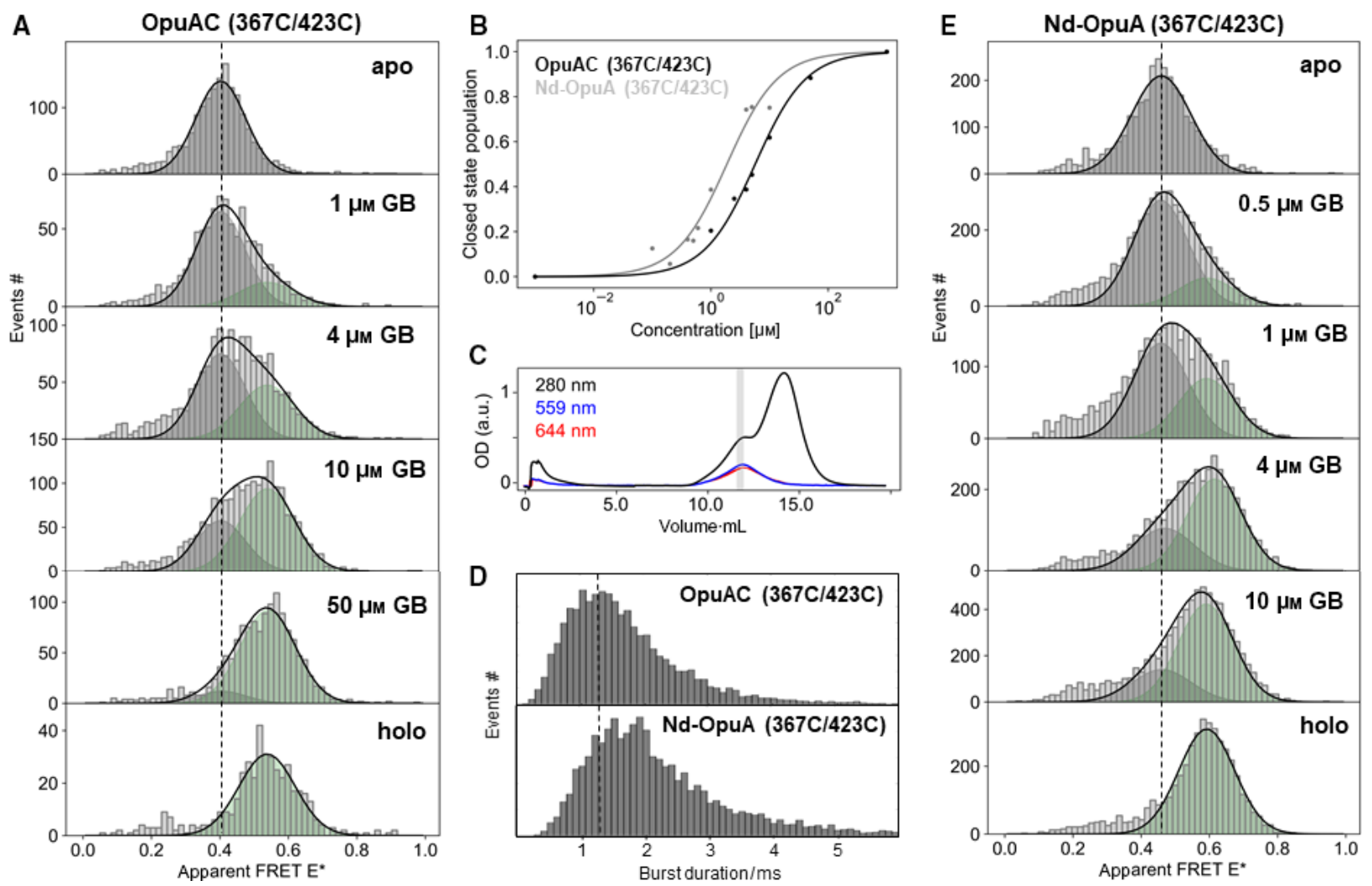

Fig. 3. smFRET studies of intramolecular SBD conformation of free OpuAC or in the context of the transporter embedded in nanodiscs. (A, E). Apparent FRET efficiency histogram of OpuAC/Nd-OpuA (367C/423C) at different glycine betaine concentrations [GB] as indicated in the figure. The relative populations of open/apo and holo/closed state were determined using a double-gaussian fitting model with a fixed mean and width and were plotted in (B) as a function of ligand concentration. (C) Size-exclusion chromatogram of fluorophore-labelled Nd-OpuA (367C/423C), light grey bar annotates the fraction used for the smFRET experiments. Examples of the full data sets are shown in Fig. S2/ S3. (D) Differences in burst duration, related to size increase in Nd-OpuA, were determined from the example data sets of panel (A, E).

difference in $K_{\mathrm{d}}$ can be seen by distinct ratios of open/ closed-state population at similar concentrations of glycine betaine (Fig. 3A vs. E), an observation that is in line with a proposed $K_{\mathrm{d}}$ increase for OpuAC in the context of the transporter from Ref. [54]. We can thus conclude that the conformational changes in OpuAC and ligand binding are slightly influenced by its linkage with the translocator domain of OpuA.

In type I $\mathrm{ABC}$ importers, TMDs are known to transit from the inward-facing state ('resting', free or ADP-bound) to the outward-facing ATP-bound state during transport [55]. To understand how these conformational changes are transmitted to the SBDs, we monitored the conformational states of OpuAC within the nanodisc-embedded OpuA (Fig. 5A, Fig. S6).

Our data suggest that addition of ATP does not influence the conformational state of OpuAC either in the absence or presence of glycine betaine, using variant Nd-OpuA (367C/423C) (Fig. 5A). Note that we assume here that ATP hydrolysis is slow at room temperature and can be used to induce conformational switching in $\mathrm{ABC}$ transporters using ALEX microscopy as shown previously for McjD [23]. This idea was supported by the fact that we obtained identical results as shown in Fig. 5 (+ATP) with AMPPNP (Fig. S6b). We thus conclude that the conformational changes of the TMDs driven by ATP binding are not transmitted to the SBDs in a way detectable by our FRET assay. However, we must note that the SBD-labelling scheme we used might impair the docking of OpuAC to the TMDs. Thus, future studies should focus on heterodimer expression of OpuA to avoid the presence of more than one donor-acceptor pair and consider backside labelling of OpuAC to avoid such complications (see Discussion for details).

\section{The conformational arrangement of membrane- anchored SBDs}

We next used the established fluorophore labelling procedure of nanodisc-reconstituted OpuA to study relative changes in interdomain distances between the 

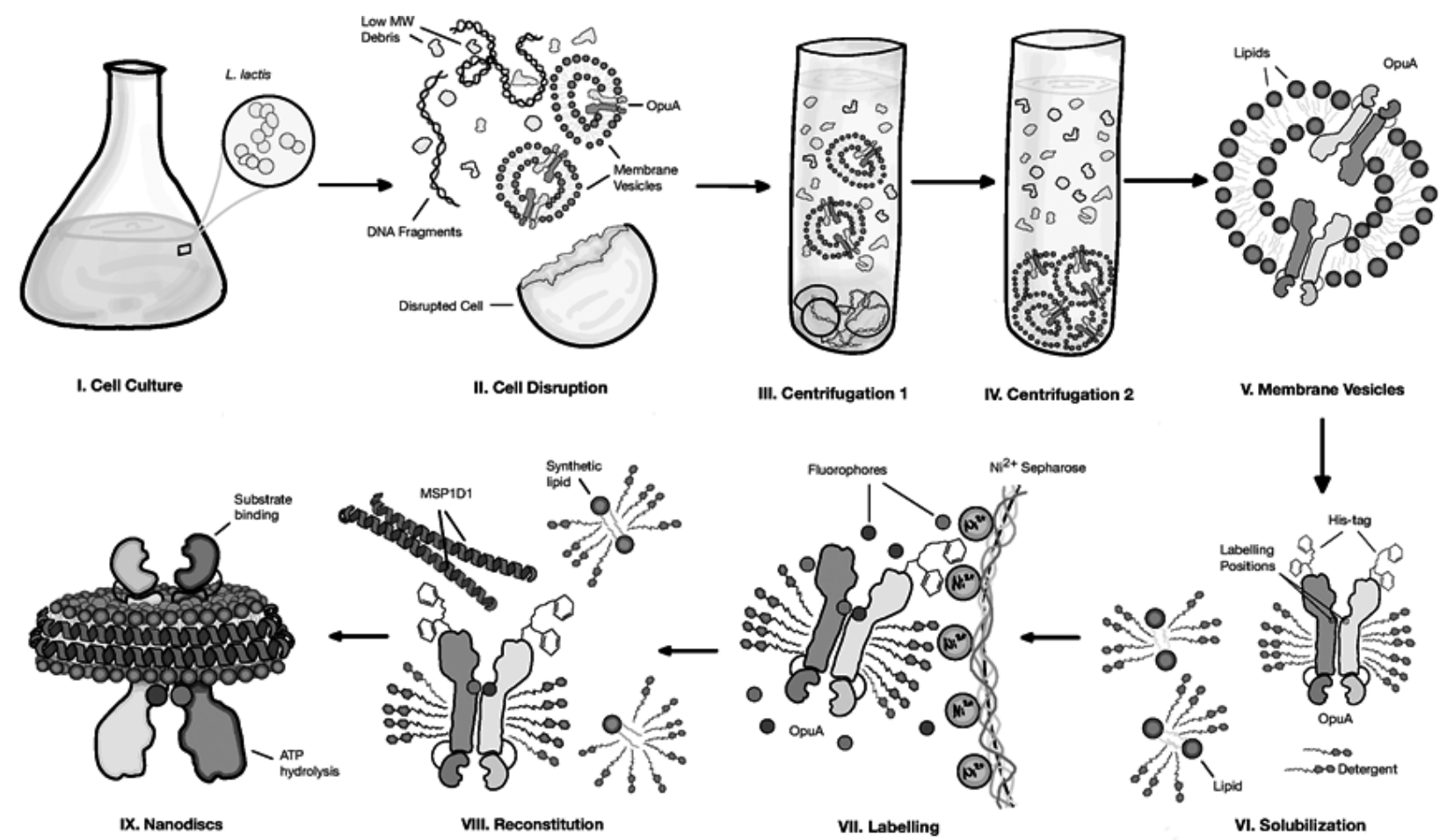

Fig. 4. Workflow for labelling of nanodisc-reconstituted OpuA for smFRET studies. The OpuA cysteine derivatives (example used here refer to TMD positions) were overexpressed in Lactococcus Lactis (step I). The cells were mechanically ruptured (step II), and transportercontaining membrane vesicles were isolated (steps III-IV). At this stage, the membrane vesicles can be stored at $-80^{\circ} \mathrm{C}$ until needed for a period of 6-12 months. OpuA was released from membrane vesicles with DDM (step VI). The solubilized transporter complex was then purified and labelled utilizing affinity chromatography (step VII). In the final step, OpuA was reconstituted into nanodiscs using synthetic lipids and an amphipathic scaffold protein $\mathrm{MSP}_{1} \mathrm{D}_{1}$ [66] (step VIII).
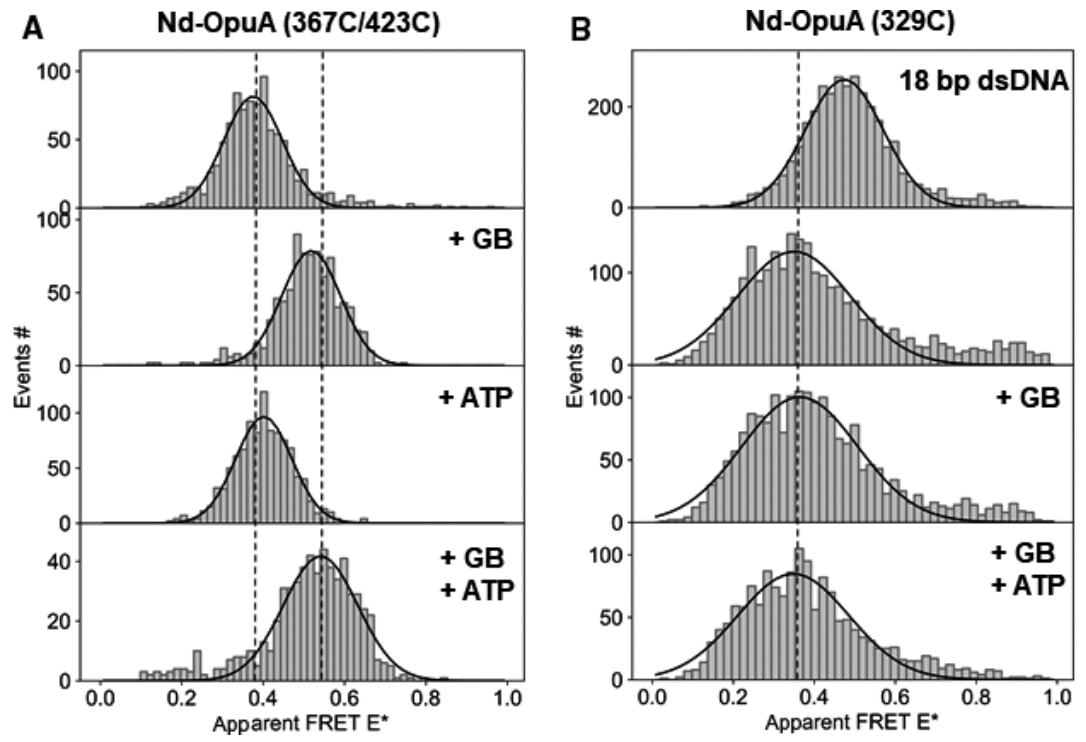

Fig. 5. ATP- and ligand-dependent conformational switching of OpuA in nanodiscs with intramolecular SBD labels. (A) Nd-OpuA (367C-423C) and interdomain labels. (B) Nd-OpuA (329C).

two SBD domains within the full transporter (Fig. 1C). For this, the labelling scheme was tailored to maintain only one cysteine per SBD and thus two per full transporter, that is Nd-OpuA (329C). The FRET efficiency histograms of Nd-OpuA (329C) were obtained in the apo state or in the presence of glycine 
betaine and/or ATP (Fig. 5B). The FRET efficiency distributions were wider as compared to a static DNA sample with one defined distance or conformational state (Fig. 5B). Similar observations were also made with another labelling positions, that is $\mathrm{Nd}-\mathrm{OpuA}$ (440C) and Nd-OpuA (458C) displayed in Fig. S6c. In comparison with the dsDNA sample, this suggests that either fast distance fluctuations occur on the sub-millisecond timescale or reveal the existence of multiple distances that give rise to overlapping distributions. To rationalize these interpretations, that is the existence of more than one possible structural state or a larger ensemble of states, we analysed a static DNA sample (Fig. 5B, upper panel). Here, the donor-acceptor distance is $18 \mathrm{bp}$ suggesting $6.1 \mathrm{~nm}$ distance between donor-acceptor attachment points using a linear DNA model and $7.2 \mathrm{~nm}$ for cylindrical DNA model [56]. As was shown previously, the width of such a distribution depends solely on photon statistics (and background) and is characteristic of a static conformational state [31,57]. The broad and nonspecific FRET efficiency distributions of the interdomain labels in Nd-OpuA (329C) can also explain the lower data quality of $\mathrm{Nd}$ OpuA $(367 \mathrm{C} / 423 \mathrm{C})$ in comparison with soluble OpuAC (367C/423C). Nd-OpuA (367C/423C) showed a significantly elevated number of unspecific FRET events, which were likely related to interdomain FRET events and were found around the major population at $\mathrm{E}^{*} \sim 0.4$ of intramolecular FRET within OpuAC (see Fig. S2, line 1 vs. line 2) due to the possibility of labelling both SBDs.

\section{Towards smFRET studies of the TMDs}

As high-resolution structures of the translocator domain (TMD) of OpuA were not available to us, we performed homology modelling by using the SwissModel server [48,49] (Note S1 and Figs S7 and S8) to identify suitable positions in the TMD for smFRET studies. The best templates for modelling were the structures of the bacterial importers for maltose (4JBW), molybdate tungstate (2ONK), methionine (3DHW) and molybdate sulfate (3D31). Unfortunately, the sequence identity was $<26 \%$ and the sequence similarity was $<32 \%$, suggesting that the resulting model may not be entirely accurate.

Keeping the limitations of the model and its unclear accuracy in mind, we focussed here on establishing an experimental pipeline to identify suitable TMD residues for smFRET with the goal to visualize changes between the inward- and outward-facing conformation. As a first step, we identified nonconserved residues that were surface-exposed and then residues that undergo significant distance changes between the inward- and outward-facing conformation in the model (Figs S7 and S8). Because our choices were based on a homology model, we decided to generate a larger number of cysteine derivatives (Figs 6 and 7A). The translocator domain of OpuA is homodimeric, and so only a single cysteine substitution per protomer would be sufficient to obtain two anchor points for fluorophores. The resulting derivatives were subsequently tested for their in vivo/in vitro functionality and labelling efficiency (Fig. 7A).

The selected TMD derivatives were labelled with different fluorophore pairs as exemplified in Fig. 6 for Nd-OpuA (176C) with TMR/Cy5 and for Nd-OpuA (12C) with Alexa 555/Alexa 647. As shown in Fig. 6, wild-type OpuA was not labelled with maleimide fluorophores due to the absence of thiol groups. The labelling efficiencies were derived by monitoring the absorbance of the protein and the fluorescent dyes at their corresponding maximum wavelength. Concentration determination of protein and fluorophores was performed using the Lambert-Beer law and published extinction coefficients (see Methods section and Table S1). Unfortunately, the contribution of $\mathrm{MSP}_{1} \mathrm{D}_{1}$ to the $280 \mathrm{~nm}$ absorbance could not be deconvoluted, because methods to perform such a deconvolution only became available quite recently [58]. The labelled fraction $(\sim 11-12 \mathrm{~mL})$ expected to contain the two OpuA components in equimolar ratio was selected for subsequent testing in biochemical and biophysical assays (Fig. 6C,D).

To test the effect of the cysteine mutations on function, we first performed in vivo uptake assays using radiolabelled glycine betaine (Fig. 7A) as described in Ref. [40]. Moreover, to evaluate the effect of fluorophore attachment, we determined ATP hydrolysis rates for OpuA (12C) labelled with TMR-Cy5 pair in comparison with OpuA wild-type. In Fig. 7A, we summarize the relevant, substrate-stimulated ATPase activities relative to wild-type; full data sets of different biochemical conditions (apo, $\mathrm{KCl}, \mathrm{GB}$ and $\mathrm{KCl}$ ) are shown in Fig. S6. We identified the OpuA (12C) derivative as a promising candidate with a good labelling efficiency ( $\sim 40 \%$ total labelling efficiency of donor and acceptor dye) and $\sim 70 \%$ substrate-stimulated ATPase activity when labelled.

We performed ALEX experiments on the apo-protein state of OpuA (12C) under ATP- and ligand-free conditions in both detergent and nanodiscs (Fig. 7C). Discrepancies were observed between labelling efficiency of OpuA in detergent and labelling efficiency of OpuA in nanodiscs (Fig. 7C) due to the biobeads interacting with the fluorophores (Fig. S9, Methods: 

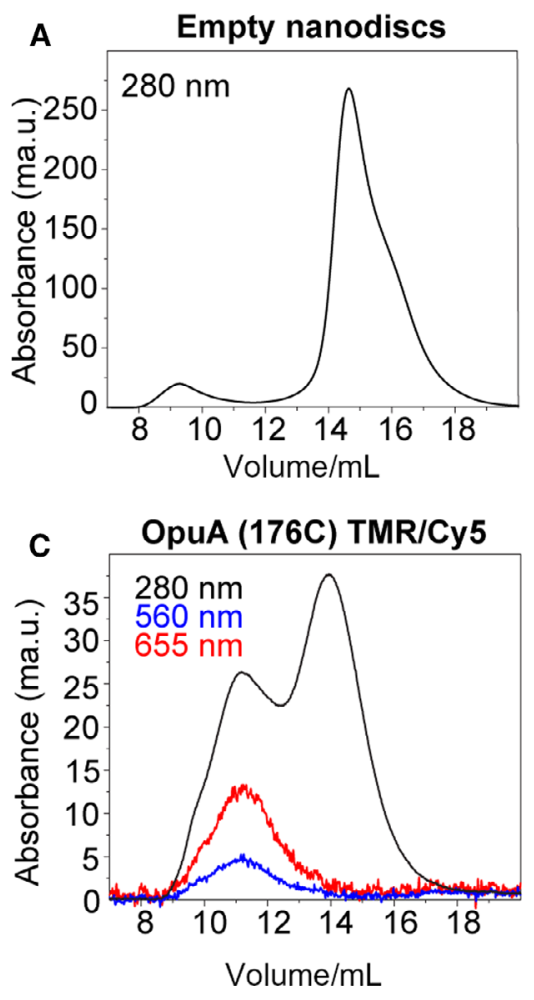
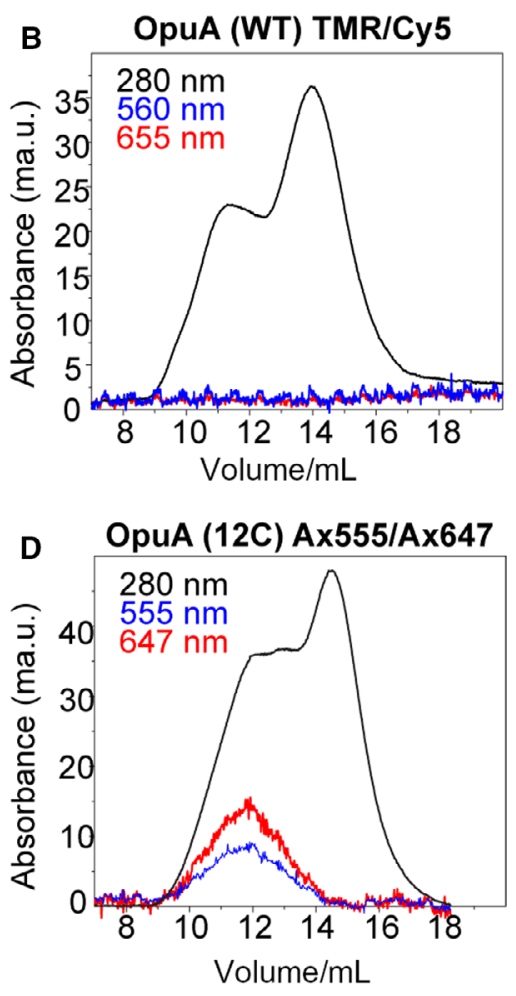

Fig. 6. Optimization of fluorophore labelling for smFRET studies of OpuA TMD mutants. (A) Size-exclusion chromatograms of (I) reconstituted nanodiscs without any OpuA. (B) Wildtype OpuA reconstituted in nanodiscs and labelled with TMR and Cy5 to evaluate the possibility of nonspecific binding of the fluorophores to the cysteine-less $\mathrm{Nd}$ OpuA. (C) Nd-OpuA 176C labelled with TMR and Cy5. (D) Nd-OpuA 12C labelled with Alexa 555 and Alexa 647. Al nanodisc preparations show a shoulder at lower elution volumes, which might indicate nanodiscs with more than one transporter.
Fluorophore labelling of OpuA). For nanodisc-reconstituted OpuA, the labelling efficiency was $\sim 40 \%$ for TMR-Cy5 (Fig. 6C), the donor-acceptor fraction was expected to be $<10 \%$. It is likely for this reason that in our preliminary ALEX experiments, we observed a high degree of random coincidence, that is coincident detection of donor- and acceptor-only species due to the high concentrations needed to see FRET events. This random coincidence was observed as a smear from low $\mathrm{E} /$ high $\mathrm{S}$ to low $\mathrm{S}$ values in this data set (Fig. 7C, 1-shaped lines between donor- and acceptoronly at low FRET efficiency). Nevertheless, for TMRCy5-labelled protein, there was a clear species at $E^{*} \sim 0.7$. The presence of low-FRET species representing a more open conformational state of the protein hidden by the high level of random coincidence cannot be ruled out. All other cysteine variants (Fig. 7A) gave smFRET results with still smaller donor-acceptor yields and thus no interpretable FRET efficiency histograms.

We next tried to use a commonly employed fluorophore pair Alexa Fluor 555-647 and obtained labelling efficiencies of $39 \%$ in nanodiscs and $46 \%$ in detergent (Fig. 7C). Despite the fact that the fluorophores perform better in terms of brightness, NdOpuA (12C) labelling with Alexa dyes resulted in noisy data in both nanodises and detergent. Based on the quality of the available data, no useful interpretation was possible. We attribute the poor quality of the resulting histograms to artefactual interactions of the dyes with the protein; for example, the aggregation of OpuA in detergent might have occurred and could explain why higher yields of donor-acceptor-containing molecules found in the detergent solution in comparison with lipid nanodiscs (Fig. 7C). This example shows clearly that better data quality is required to rule out artefacts and the availability of a high-resolution structure would facilitate assay design and final interpretation of smFRET results in a meaningful way.

\section{Discussion and Conclusion}

Long-distance allosteric communication is central to the function of $\mathrm{ABC}$ importers [59] and exporters [60]. The association and dissociation events of the NBDs that are driven by ATP binding and hydrolysis are transmitted to TMDs, which undergo conformational rearrangements that facilitate transport. SBDs act as primary receptors to bind and donate their respective substrates for transport initiation. The tight connectivity between the different domains is undisputed, and its functional repercussions have been verified in many systems, for example the means by which the 


\begin{tabular}{ccccc}
\hline $\begin{array}{c}\text { A } \begin{array}{c}\text { OpuA TMD- } \\
\text { Mutant }\end{array} \\
\text { (\%); unlabeled }\end{array}$ & $\begin{array}{c}\text { Relative in- } \\
\text { vivo uptake } \\
\text { (\%) }\end{array}$ & $\begin{array}{c}\text { Relative in vitro } \\
\text { ATPase assay } \\
\text { (labelled, \%) }\end{array}$ & $\begin{array}{c}\text { Labelling } \\
\text { Efficiency with } \\
\text { TMR-Cy5 pair (\%) }\end{array}$ & $\begin{array}{c}\text { Relative } \\
\text { distance } \\
\text { change }(\AA)\end{array}$ \\
\hline Ala 12 Cys & 63 & 70 & 39 & 10.7 \\
Trp 14 Cys & 61 & 43 & 27 & 10 \\
Leu 102 Cys & 60 & 46 & 4 & 8.4 \\
Leu 106 Cys & 66 & 96 & 7 & 10.5 \\
Ile 110 Cys & 83 & 120 & 7 & 8.3 \\
Asn 176 Cys & 84 & 16 & 49 & 8.4 \\
Arg 180 Cys & 130 & 25 & 43 & 10 \\
Gln 181 Cys & 98 & 13 & 18 & 9.7 \\
Ser 183 Cys & 117 & 48 & 30 & 14.4 \\
Thr 184 Cys & 89 & 28 & 41 & 12.4 \\
Thr 196 Cys & 0 & 8 & 81 & 12.3 \\
Arg 198 Cys & 5 & - & - & 12.8 \\
Leu 244 Cys & 8 & - & - & 14 \\
Ala 245 Cys & 105 & 49 & 37 & 7.9 \\
\hline
\end{tabular}

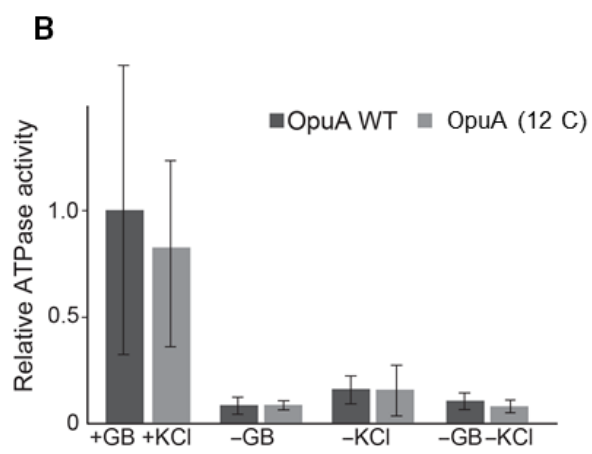

C Nd-OpuA(12 C): TMR-Cy5

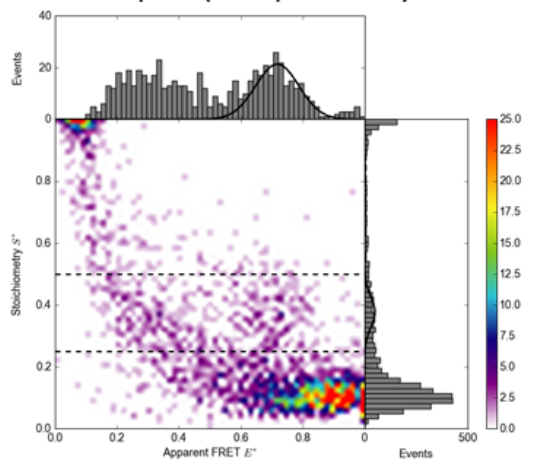

Nd-OpuA (12 C): Alexa555-647

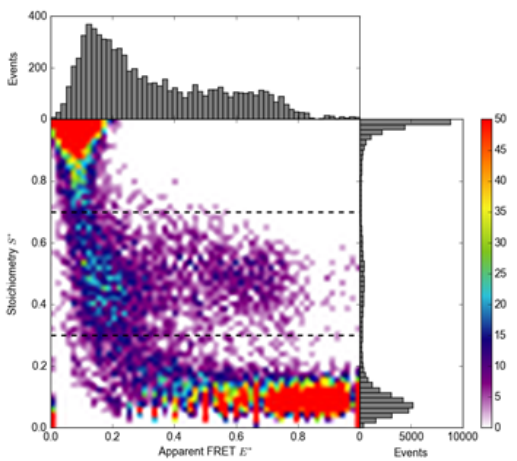

DDM-OpuA (12 C): Alexa555-647

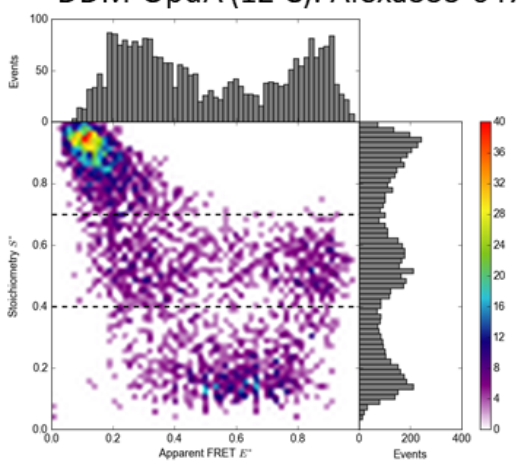

Fig. 7. smFRET studies of TMD-labelled OpuA with OpuA (12C). (A) To select the appropriate cysteine derivatives for smFRET experiments, we tested the effect of the cysteine point mutations and the fluorophore labelling on the activity of OpuA. Labelling efficiency was calculated by considering both donor- and acceptor-labelling yields from SEC runs when comparing protein and dye absorbance. In the last column, we also present the distance change between the modelled inward and outward conformation, an important parameter for the sensitivity of the smFRET assay. (B) ATPase activity comparison between labelled Nd-OpuA (12C) and OpuA wild-type (WT); mean \pm SEM is shown $(N=3)$. (C) Nd-OpuA (12C) with different fluorophore pairs showing increased donor-acceptor yield for use of Alexa 555-647. For the data, all photon burst search was used $(M=15, T=500 \mu$ s and $L=50)$ with additional thresholding of all photons $>100$. The S-range for 1D-E* histograms is indicated in the figure as dashed line.

conformational changes of SBDs dictate substrate specificity [21] and transport [19]. However, many salient features of these interdomain associations remain elusive and the latter form the basis of the distinction between different transporter systems.

Substrate capture by SBDs is the initial step for transport. In type I systems, the SBDs alternate between two distinct conformations, an open-unliganded form in which the two lobes resemble an open book, and a closed-liganded one, in which the ligand is captured by the well-known Venus fly trap motion [61]. In contrast, in type II importers the structural rearrangements in the SBDs driven by the ligand are minor [61]. OpuAC from L. lactis is a type I SBD linked to the TMDs. Thus, two OpuAC domains are present per functional transporter. In this paper, the SBD OpuAC was labelled with donor and acceptor fluorophores and its conformational states were probed by smFRET. Soluble OpuAC, as also previously reported for other members of the periplasmic binding protein family [21], binds its substrate by the Venus fly trap motion and most likely an induced-fit mechanism according to our data. Its structural transitions are ligand-dependent, and by titrating the ligand, we derived $K_{\mathrm{d}}$-values by kinetic rate analysis and occupation ratios of the conformational states. These values are in excellent agreement with the ones determined in bulk, indicating that labelling and our smFRET assays do not interfere with ligand binding.

We also probed the conformational changes of OpuAC within the full transporter complex. For this, we tested many distinct labelling protocols including fluorophore addition (a) during the purification, (b) solubilization or (c) reconstitution of the entire transporter. The highest labelling efficiency could be achieved when the fluorescent dyes were added to 
detergent-solubilized OpuA that was immobilized on the Ni-NTA column material (Fig. 4). The 2D ALEX plot of the labelled OpuAC within OpuA indicated the existence of multiple labelling populations, as expected because of the two OpuAC domains per transporter, and the possibility of attachment of two fluorescent labels per OpuAC. With analysis based on fluorophore brightness (Fig. S3), we can separate the populations (Figs 1A and 3). The results indicate that OpuAC within the entire transporter samples similar conformation states: open-unliganded and closed-liganded state. Furthermore, ligand titrations indicated that OpuAC within OpuA might have elevated binding affinity for glycine betaine. We must note that currently we cannot exclude the labels that hinder the docking process and further optimization of the labelling scheme is required to minimize this possibility.

The second step of transport is represented by the interaction of the SBD with the TMDs. In type I importers, the TMDs differentiate between the openunliganded and the closed-liganded state of the SBD. It is believed that interaction of the closed-liganded state with the TMD [19] efficiently triggers ATP hydrolysis [42,62]. Conversely, the ATPase activity of type II importers can be ligand-independent or shows at least much smaller stimulation values in the presence of liganded SBD/SBP $[63,64]$. Such findings suggest a communication of the SBDs and the NBDs via the TMDs. Within our assays, we probed the OpuAC conformational states during transport conditions (glycine betaine + ATP) and throughout its resting state (apo). Addition of ATP and glycine betaine had no effect on the conformational dynamics of OpuAC. Since a single SBD interacts with the TMD to deliver one substrate per transport cycle [42], an important question is whether the two OpuAC domains interact stochastically or in a concerted fashion with the TMD to release the substrate. To probe the relative movement of the two OpuAC domains, we placed one probe per domain using three different OpuA derivatives (329C, 440C and 458C). Our results indicate that probably there are fast, nonconcerted motions between the two SBDs that are independent of the state of the TMDs, since we did not find changes in the distribution upon addition of ATP/GB. All three mutants also gave fairly broad featureless distributions (Fig. 5B, Fig. S6c), which might relate to uncorrelated stochastic motion. Future studies should reveal the relative motions of the two OpuAC domains during nucleotide cycling in more detail using advanced fluorescence methods with improved temporal resolution such as multiparameter fluorescence detection (MFD analysis [30]), fluorescence correlation spectroscopy or pulsed-interleave excitation [57].

The absence of ATP/ligand-driven effects in our study might, however, also result from a failure of our labelled mutants to dock to the translocator TMDs due to steric hindrance effects and the chosen positions, which were only optimized for smFRET and not the biochemical activity. In future experiments, such effects must be avoided by use of alternative labelling schemes, for example on the back of OpuAC. Optimal labelling residues on the back of OpuAC (hinge region) for use of $\mathrm{Cy} 3 \mathrm{~B} / \mathrm{ATTO} 647 \mathrm{~N}$ or Alexa $555 / 647$ would be A414C-A569C, A414C-A565C, A414CA570C or A414C-A562C for intradomain monitoring (or any of these single cysteines for interdomain monitoring) considering the Förster radii of the two fluorophore pairs and the fact that they were used successfully for labelling nanodisc-reconstituted OpuA here.

The next step of transport involves the TMD conformational changes that need to occur to promote the passage of the substrate to the cell interior. Those changes are assumed to be strongly coupled to the ATPase cycle in type I importers [59]. Type I ABC transporters switch from the resting state of the inward-facing conformation to the outward, a process coupled to ATP and SBD binding events - which is distinct for type II importers. That might be the reason that in type I importers, the ATPase activity is better regulated (i.e. here ATP hydrolysis is generally well coupled to translocation), whereas type II importers manifest high basal ATPase activities in the absence of SBD and substrate. To probe transitions between the inward- and outward-facing conformation of OpuA, we produced a large number of cysteine derivatives. We started with a set of 14 mutants of which 11 still proved to be functional $(>60 \%$ transport activity in vivo). Remarkably, while the chosen positions are surface-exposed according to our homology model, the majority could not be labelled well with dyes, suggesting that the position might be distinct from what is suggested in the model. The low sequence conservation of OpuA with available structures renders this finding not surprising. However, we selected the best derivative with respect to the labelling efficiency and the retention of the in vivo and in vitro functionality. The interpretations of the smFRET results of Nd-OpuA (12C) are still not fully clear since the resulting donor-acceptor yields were too low for in-depth interpretation. Without further studies, we cannot claim with confidence whether we observe one or multiple conformational states at this position and also whether the choice of different fluorophore pairs 
might have impacted our observations (Fig. 7C). In the future, more experiments with improvements on labelling efficiency and in particular donor-acceptorcontaining functional transporter molecules are required. This type of problem, that is high labelling efficiency yet low donor-acceptor fractions when labelling TMD residues in $\mathrm{ABC}$ transporters, was reported by us [23] and others [24] before. This complication was much less pronounced when the labelling was performed on the NBDs [23]. Improvement of this aspect could reduce the measurement times from $>1 \mathrm{~h}$ for proper statistics, too much shorter periods, and would allow further testing of different biochemical studies of the transport cycles.

After our manuscript was accepted for publication, a paper was published reporting the structure of the OpuA transporter in different conformational states [65]. The new structural information allowed further interpretations of our results: (a) The structure of OpuA was obtained in different conformations (PDBs: 7AHC, 7AHD, 7AHE, 7AHH) and revealed a unique TMD 'scaffold' [65]. The latter comprises residues 190 with two amphipathic helices that lie on top of the outer membrane leaflet, where two transmembrane helices act as membrane 'anchors'. The structure revealed that our homology model (Fig. S7) was incorrect with respect to the N-terminal part of OpuA. However, most of the residues that we selected to probe conformational changes on the translocator (Fig. 7A) were indeed facing either the cytoplasmic or the periplasmic side. Only three out of the fourteen selected residues were 'true' transmembrane residues (Leu102, Leu106 and Leu110). Noteworthy, in our unbiased experiments, we noticed that the labelling efficiency of the corresponding membrane residues was extremely low $(<10 \%)$ in comparison with all others (> 40\%). Importantly, (b) the cysteines in OpuAC (367C/423C) employed to compare the SBD conformations between a free or a membrane-linked SBD (Fig. 3) turned out to be distant from the translocator domain (PDBs: 7AHD, 7AHH). Thus, dye attachment at these positions is unlikely to interfere with SBD docking. (c) In the apo-protein state of the transporter (PDB: 7AHC), both SBDs are not resolved, most likely due to structural flexibility as also suggested by Sikkeman et al. [65]. This is also in line with our interpretations in Fig. 5B (upper panel), in which we directly probed fast dynamics between the SBDs. Very importantly though, (d) our data suggest that the SBDs do not dock stably onto the TMD for all conditions tested (Fig. 5: addition of ATP, glycine betaine or both). To achieve stable SBD docking onto the TMDs, Sikkeman et al. introduced OpuA (E190Q) derivative (PDB: 7AHD) or the addition of cyclic-diAMP (PDB: 7AHH). This recent structural work suggests that SBD fluorophore labelling as used here might actually not impair with SBD-TMD docking and our interpretations based on data in Figs 3 and 5 might be physiologically relevant.

In summary, despite all problems encountered, we here pave the way for future experimental smFRET studies that aim at understanding the salient features of inter- and intradomain communication in $\mathrm{ABC}$ transporters and in particular for OpuA.

\section{Acknowledgements}

This work was financed by an NWO Veni grant (722.012.012 to GG), an ERC Starting Grant (ERCSTG 638536 - SM-IMPORT to TC) and the Zernike Institute for Advanced Materials (support to GG and TC). GG also acknowledges an EMBO fellowship (long-term fellowship ALF 47-2012), and the Rega Foundation Postdoctoral Programme of KU Leuven. We thank J. Hammerl for preparation of Fig. 4 and C. Gebhardt for providing data analysis scripts. We thank B. Poolman for continuous support of the project, which included conceptional discussions on the structure and function of OpuA and access to materials and protocols. We finally thank G. K. SchuurmanWolters for experimental support, and B. Poolman and D. A. Griffith for critically reading the manuscript.

\section{Author contributions}

GG and TC conceived and designed the study and supervised the project. KT, RV, MK and GG performed molecular biology and biochemistry studies. $\mathrm{RV}$ and GG developed the labelling protocols. KT, $\mathrm{RV}, \mathrm{MB}$ and $\mathrm{GG}$ performed single-molecule experiments. KT, MB, GG and TC analysed data. KT, GG and TC prepared figures and wrote the manuscript. All authors contributed to discussion of the research and approved the final version of the manuscript.

\section{References}

1 Schuurman-Wolters GK and Poolman B (2005) Substrate specificity and ionic regulation of GlnPQ from Lactococcus lactis. An ATP-binding cassette transporter with four extracytoplasmic substrate-binding domains. Biol Chem 280, 23785-23790.

2 Sharom FJ (2008) ABC multidrug transporters: structure, function and role in chemoresistance. Pharmacogenomics 9, 105-127. 
3 Abele R and Tampe R (2004) The ABCs of immunology: structure and function of TAP, the transporter associated with antigen processing. Physiology (Bethesda) 19, 216-224.

4 Biemans-Oldehinkel E, Doeven MK and Poolman B (2006) ABC transporter architecture and regulatory roles of accessory domains. FEBS Lett 580, 1023-1035.

5 Davidson AL, Dassa E, Orelle C and Chen J (2008) Structure, function, and evolution of bacterial ATPbinding cassette systems. Microbiol Mol Biol Rev 72, 317-364, table of contents.

6 Oldham ML, Davidson AL and Chen J (2008) Structural insights into ABC transporter mechanism. Curr Opin Struct Biol 18, 726-733.

7 Kos V and Ford RC (2009) The ATP-binding cassette family: a structural perspective. Cell Mol Life Sci 66, 3111-3126.

8 Parcej D and Tampe R (2010) ABC proteins in antigen translocation and viral inhibition. Nat Chem Biol 6, $572-580$.

9 Locher KP (2009) Review. Structure and mechanism of ATP-binding cassette transporters. Philos Trans $R$ Soc Lond B Biol Sci 364, 239-245.

10 Plumptre CD, Eijkelkamp BA, Morey JR, Behr F, Couñago RM, Ogunniyi AD, Kobe B, O'Mara ML, Paton JC and McDevitt CA (2014) AdcA and AdcAII employ distinct zinc acquisition mechanisms and contribute additively to zinc homeostasis in Streptococcus pneumoniae. Mol Microbiol 91, 834-851.

11 Riordan JR (2008) CFTR function and prospects for therapy. Annu Rev Biochem 77, 701-726.

12 Teague SJ (2003) Implications of protein flexibility for drug discovery. Nat Rev Drug Discovery 2, 527-541.

13 Majumder P, Mallela AK and Penmatsa A (2018) Transporters through the looking glass: an insight into the mechanisms of ion-coupled transport and methods that help reveal them. J Indian Inst Sci 98, 283-300.

14 Majumdar DS, Smirnova I, Kasho V, Nir E, Kong X, Weiss S and Kaback HR (2007) Single-molecule FRET reveals sugar-induced conformational dynamics in LacY. Proc Natl Acad Sci USA 104, 12640-12645.

15 Zhao Y, Terry D, Shi L, Weinstein H, Blanchard SC and Javitch JA (2010) Single-molecule dynamics of gating in a neurotransmitter transporter homologue. Nature 465, 188-193.

16 Zhao Y, Terry DS, Shi L, Quick M, Weinstein H, Blanchard SC and Javitch JA (2011) Substratemodulated gating dynamics in a $\mathrm{Na}+$-coupled neurotransmitter transporter homologue. Nature 474, 109-113.

17 Akyuz N, Altman RB, Blanchard SC and Boudker O (2013) Transport dynamics in a glutamate transporter homologue. Nature 502, 114-118.

18 Erkens GB, Hanelt I, Goudsmits JM, Slotboom DJ and van Oijen AM (2013) Unsynchronised subunit motion in single trimeric sodium-coupled aspartate

transporters. Nature 502, 119-123.

19 Gouridis G, Schuurman-Wolters GK, Ploetz E, Husada F, Vietrov R, de Boer M, Cordes T and Poolman B (2015) Conformational dynamics in substrate-binding domains influences transport in the ABC importer GlnPQ. Nat Struct Mol Biol 22, 57-64.

20 van der Velde JH, Oelerich J, Huang J, Smit JH, Aminian Jazi A, Galiani S, Kolmakov K, Guoridis G, Eggeling C, Herrmann A et al. (2016) A simple and versatile design concept for fluorophore derivatives with intramolecular photostabilization. Nat Commun 7, 10144.

21 de Boer M, Gouridis G, Vietrov R, Begg SL, Schuurman-Wolters GK, Husada F, Eleftheriadis N, Poolman B, McDevitt CA and Cordes T (2019) Conformational and dynamic plasticity in substratebinding proteins underlies selective transport in $\mathrm{ABC}$ importers. Elife 8, e44652.

22 Jazi AA, Ploetz E, Arizki M, Dhandayuthapani B, Waclawska I, Krämer R, Ziegler C and Cordes T (2017) Caging and photoactivation in single-molecule Förster resonance energy transfer experiments. Biochemistry 56, 2031-2041.

23 Husada F, Bountra K, Tassis K, de Boer M, Romano M, Rebuffat S, Beis K and Cordes T (2018) Conformational dynamics of the ABC transporter McjD seen by single-molecule FRET. EMBO J 37, e100056.

24 Yang M, Livnat Levanon N, Acar B, Aykac Fas B, Masrati G, Rose J, Ben-Tal N, Haliloglu T, Zhao Y and Lewinson $O$ (2018) Single-molecule probing of the conformational homogeneity of the $\mathrm{ABC}$ transporter BtuCD. Nat Chem Biol 14, 715-722.

25 Liu Y, Liu Y, He L, Zhao Y and Zhang XC (2018) Single-molecule fluorescence studies on the conformational change of the ABC transporter MsbA. Biophys Rep 4, 153-165.

26 Ha T, Enderle T, Ogletree D, Chemla DS, Selvin PR and Weiss S (1996) Probing the interaction between two single molecules: fluorescence resonance energy transfer between a single donor and a single acceptor. Proc Natl Acad Sci USA 93, 6264-6268.

27 Ha T (2001) Single-molecule FRET. Single Mol 2, 283 284.

28 Lerner E, Cordes T, Ingargiola A, Alhadid Y, Chung S, Michalet X and Weiss S (2018) Toward dynamic structural biology: two decades of single-molecule Förster resonance energy transfer. Science 359, eaan1133. https://doi.org/10.1126/science.aan1133

29 Muschielok A, Andrecka J, Jawhari A, Bruckner F, Cramer P and Michaelis J (2008) A nanopositioning system for macromolecular structural analysis. Nat Methods 5, 965-971.

30 Kalinin S, Peulen T, Sindbert S, Rothwell PJ, Berger S, Restle T, Goody RS, Gohlke H and Seidel CA (2012) 
A toolkit and benchmark study for FRET-restrained high-precision structural modeling. Nat Methods $\mathbf{9}$, 1218-1225.

31 Hellenkamp B, Schmid S, Doroshenko O, Opanasyuk O, Kühnemuth R, Adariani SR, Ambrose B, Aznauryan M, Barth A and Birkedal V (2018) Precision and accuracy of single-molecule FRET measurementsa multi-laboratory benchmark study. Nat Methods 15, 669-676.

32 Cordes T, Santoso Y, Tomescu AI, Gryte K, Hwang LC, Camará B, Wigneshweraraj S and Kapanidis AN (2010) Sensing DNA opening in transcription using quenchable Forster resonance energy transfer. Biochemistry 49, 9171-9180.

33 Kapanidis AN, Lee NK, Laurence TA, Doose S, Margeat E and Weiss S (2004) Fluorescence-aided molecule sorting: analysis of structure and interactions by alternating-laser excitation of single molecules. Proc Natl Acad Sci USA 101, 8936-8941.

34 Robb NC, Cordes T, Hwang LC, Gryte K, Duchi D, Craggs TD, Santoso Y, Weiss S, Ebright RH and Kapanidis AN (2013) The transcription bubble of the RNA polymerase-promoter open complex exhibits conformational heterogeneity and millisecond-scale dynamics: implications for transcription start-site selection. J Mol Biol 425, 875-885.

35 Gouridis G, Hetzert B, Kiosze-Becker K, de Boer M, Heinemann H, Nurenberg-Goloub E, Cordes T and Tampe R (2019) ABCE1 controls ribosome recycling by an asymmetric dynamic conformational equilibrium. Cell Rep 28, 723-734, e6.

36 Goudsmits JM, Slotboom DJ and van Oijen AM (2017) Single-molecule visualization of conformational changes and substrate transport in the vitamin B 12 ABC importer BtuCD-F. Nat Commun 8, 1-10.

37 Ruan Y, Miyagi A, Wang X, Chami M, Boudker O and Scheuring S (2017) Direct visualization of glutamate transporter elevator mechanism by highspeed AFM. Proc Natl Acad Sci USA 114, 1584-1588.

38 Joseph B, Sikora A, Bordignon E, Jeschke G, Cafiso DS and Prisner TF (2015) Distance measurement on an endogenous membrane transporter in $E$. coli cells and native membranes using EPR spectroscopy. Angew Chem 127, 6294-6297.

39 Sahu ID, McCarrick RM, Troxel KR, Zhang R, Smith HJ, Dunagan MM, Swartz MS, Rajan PV, Kroncke BM and Sanders CR (2013) DEER EPR measurements for membrane protein structures via bifunctional spin labels and lipodisq nanoparticles. Biochemistry 52, 6627-6632.

40 Karasawa A, Swier LJ, Stuart MC, Brouwers J, Helms B and Poolman B (2013) Physicochemical factors controlling the activity and energy coupling of an ionic strength-gated ATP-binding cassette (ABC) transporter. Biol Chem 288, 29862-29871.
41 Biemans-Oldehinkel E, Mahmood NA and Poolman B (2006) A sensor for intracellular ionic strength. Proc Natl Acad Sci USA 103, 10624-10629.

42 Patzlaff JS, van der Heide T and Poolman B (2003) The ATP/substrate stoichiometry of the ATP-binding cassette (ABC) transporter OpuA. Biol Chem 278, 29546-29551.

43 van der Heide T, Stuart MC and Poolman B (2001) On the osmotic signal and osmosensing mechanism of an ABC transport system for glycine betaine. EMBO J 20, 7022-7032.

44 Fulyani F, Schuurman-Wolters GK, Slotboom D-J and Poolman B (2016) Relative rates of amino acid Import via the $\mathrm{ABC}$ transporter GlnPQ determine the growth performance of Lactococcus lactis. J Bacteriol 198, 477485.

45 Nguyen PT, Lai JY, Lee AT, Kaiser JT and Rees DC (2018) Noncanonical role for the binding protein in substrate uptake by the MetNI methionine ATP Binding Cassette (ABC) transporter. Proc Natl Acad Sci USA 115, E10596-E10604.

46 Gul N, Schuurman-Wolters G, Karasawa A and Poolman B (2012) Functional characterization of amphipathic $\alpha$-helix in the osmoregulatory ABC transporter OpuA. Biochemistry 51, 5142-5152.

47 Spack EG Jr, Packard B, Wier ML and Edidin M (1986) Hydrophobic adsorption chromatography to reduce nonspecific staining by rhodamine-labeled antibodies. Anal Biochem 158, 233-237.

48 Bertoni M, Kiefer F, Biasini M, Bordoli L and Schwede T (2017) Modeling protein quaternary structure of homo-and hetero-oligomers beyond binary interactions by homology. Sci Rep 7, 1-15.

49 Guex N, Peitsch MC and Schwede T (2009) Automated comparative protein structure modeling with SWISSMODEL and Swiss-PdbViewer: a historical perspective. Electrophoresis 30, S162-S173.

50 DeLano WL (2002) Pymol: an open-source molecular graphics tool. CCP4 Newslett Protein Crystallogr 40, $82-92$.

51 de Boer M, Gouridis G, Muthahari YA and Cordes T (2019) Single-molecule observation of ligand binding and conformational changes in FeuA. Biophys $J$ 117, $1642-1654$.

52 van der Velde JH, Ploetz E, Hiermaier M, Oelerich J, de Vries JW, Roelfes G and Cordes T (2013)

Mechanism of intramolecular photostabilization in selfhealing cyanine fluorophores. ChemPhysChem 14, 4084 4093.

53 Rabiner LR (1990) A tutorial on hidden markov models and selected applications in speech recognition. In Readings in Speech Recognition, pp. 267-296. Morgan Kaufmann, Los Altos, CA.

54 Karasawa A, Erkens GB, Berntsson RP, Otten R, Schuurman-Wolters GK, Mulder FA and Poolman B 
(2011) Cystathionine beta-synthase (CBS) domains 1 and 2 fulfill different roles in ionic strength sensing of the ATP-binding cassette (ABC) transporter OpuA. Biol Chem 286, 37280-37291.

55 ter Beek J, Guskov A and Slotboom DJ (2014) Structural diversity of ABC transporters. Gen Physiol 143, 419-435.

56 Le Reste L, Hohlbein J, Gryte K and Kapanidis AN (2012) Characterization of dark quencher chromophores as nonfluorescent acceptors for singlemolecule FRET. Biophys $J$ 102, 2658-2668.

57 Ploetz E, Lerner E, Husada F, Roelfs M, Chung S, Hohlbein J, Weiss S and Cordes T (2016) Förster resonance energy transfer and protein-induced fluorescence enhancement as synergetic multi-scale molecular rulers. Sci Rep 6, 33257.

58 Häusler E, Fredriksson K, Goba I, Peters C, Raltchev K, Sperl L, Steiner A, Weinkauf S and Hagn F (2020) Quantifying the insertion of membrane proteins into lipid bilayer nanodiscs using a fusion protein strategy. Biochim Biophys Acta Biomembr 1862, 183190.

59 Lewinson O and Livnat-Levanon N (2017) Mechanism of action of $\mathrm{ABC}$ importers: conservation, divergence, and physiological adaptations. J Mol Biol 429, 606-619.

60 Szöllősi D, Rose-Sperling D, Hellmich UA and Stockner T (2018) Comparison of mechanistic transport cycle models of ABC exporters. Biochim Biophys Acta Biomembr 1860, 818-832.

61 Scheepers GH, Lycklama A, Nijeholt JA and Poolman B (2016) An updated structural classification of substrate-binding proteins. FEBS Lett 590, 4393-4401.

62 Davidson AL, Shuman HA and Nikaido H (1992) Mechanism of maltose transport in Escherichia coli: transmembrane signaling by periplasmic binding proteins. Proc Natl Acad Sci USA 89, 2360-2364.

63 Borths EL, Poolman B, Hvorup RN, Locher KP and Rees DC (2005) In vitro functional characterization of BtuCD-F, the Escherichia coli ABC transporter for vitamin B12 uptake. Biochemistry 44, 16301-16309.

64 Vigonsky E, Ovcharenko E and Lewinson O (2013) Two molybdate/tungstate ABC transporters that interact very differently with their substrate binding proteins. Proc Natl Acad Sci USA 110, 5440-5445.

65 Sikkema Hendrik R, van den Noort Marco, Rheinberger Jan, de Boer Marijn, Krepel Sabrina T, Schuurman-Wolters Gea K, Paulino Cristina, Poolman Bert (2020) Gating by ionic strength and safety check by cyclic-di-AMP in the ABC transporter OpuA. Science Advances 6, eabd7697. http://dx.doi.org/10. 1126/sciadv.abd7697.
66 Kijac A, Shih AY, Nieuwkoop AJ, Schulten K, Sligar SG and Rienstra CM (2010) Lipid-protein correlations in nanoscale phospholipid bilayers determined by solidstate nuclear magnetic resonance. Biochemistry 49, 9190-9198.

\section{Supporting information}

Additional supporting information may be found online in the Supporting Information section at the end of the article.

Fig. S1. General description of smFRET experiments and analysis on the example of OpuAC (V360C/ N423C).

Fig. S2. ALEX 2D plots (as in Supplementary Figure 1) of soluble OpuAC (OpuAC 367C/423C) or nanodisc reconstituted Nd-OpuA $(367 \mathrm{C} / 423 \mathrm{C})$ labelled with Cy3B and ATTO647N fluorophores.

Fig. S3. Analysis of the labelling composition of $\mathrm{Nd}$ OpuA complexes.

Fig. S4. OpuA and MSP1D1 expression and purification.

Fig. S5. In vitro ATPase results.

Fig. S6. (a) Burst length distributions (all 100, 0.4-0.8 $\mathrm{S})$ for OpuAC (free substrate protein) and $\mathrm{Nd}-\mathrm{OpuA}$ (full transporter reconstituted in nanodiscs). (b) Additional smFRET data sets showing that AMPPNP had a similar negligible impact compared to ATP addition. (c) Additional smFRET data sets showing distributions of interdomain SBD labelling of $\mathrm{Nd}-\mathrm{OpuA}$ (458C) and Nd-OpuA (440C).

Fig. S7. The homology model of the OpuA transmembrane domain was done using Swiss-model server [3].

Fig. S8. OpuA homology models with selected labelling positions relevant to monitoring TMD movements.

Fig. S9. a. Chromatogram of unlabeled MalE. b. Chromatogram of unlabeled MalE after overnight incubation with SM2 bio-beads.

Table S1. List of maleimide fluorophores used in this study including their extinction coefficients.

Table S2. QC-PCR primers for cysteine derivatives of OpuA.

Table S3. List of crystal structures used as templates for OpuA-TMD.

Table S4. Calculated distances between the cysteine positions in the homodimer of OpuA.

Note S1. Homology model. 